Analysis of a mixed discontinuous Galerkin method for instationary Darcy flow

Peer-reviewed author version

Aizinger, Vadym; Rupp, Andreas; SCHUETZ, Jochen \& Knabner, Peter (2018) Analysis of a mixed discontinuous Galerkin method for instationary Darcy flow. In: COMPUTATIONAL GEOSCIENCES, 22(1), p. 179-194.

DOI: $10.1007 / \mathrm{s} 10596-017-9682-8$

Handle: http://hdl.handle.net/1942/23967 


\section{Computational Geosciences \\ Analysis of a mixed discontinuous Galerkin method for instationary Darcy flow --Manuscript Draft--}

Manuscript Number:

Full Title:

Article Type:

Keywords:

Corresponding Author:
COMG-D-16-00144R2

Analysis of a mixed discontinuous Galerkin method for instationary Darcy flow

Manuscript

mixed discontinuous Galerkin method; local discontinuous Galerkin method; instationary Darcy problem; stability and error analysis

Vadym Aizinger

Friedrich-Alexander-Universitat Erlangen-Nurnberg

GERMANY

\section{Corresponding Author Secondary} Information:

Corresponding Author's Institution:

Friedrich-Alexander-Universitat Erlangen-Nurnberg

Corresponding Author's Secondary Institution:

\section{First Author:}

Vadym Aizinger

First Author Secondary Information:

Order of Authors:

$$
\begin{array}{|l|}
\hline \text { Vadym Aizinger } \\
\hline \text { Andreas Rupp } \\
\hline \text { Jochen Schütz } \\
\hline \text { Peter Knabner } \\
\hline
\end{array}
$$

\section{Order of Authors Secondary Information:}

\section{Funding Information:}

\section{Abstract:}

Response to Reviewers:
We present an a priori stability and convergence analysis of a new mixed discontinuous Galerkin scheme applied to the instationary Darcy problem. The analysis accounts for a spatially and temporally varying permeability tensor in all estimates. The proposed method is stabilized using penalty terms in the primary and the flux unknowns.

\section{Reviewer 1:}

'... in the last paragraph of page 11 , the authors should be more explicit about what they call "standard" LDG, a simple sentence will do the job.':

-- We added a clarifying statement.

\section{Reviewer 2:}

1. In the interest of reproducibility of numerical work can the authors specify the time step chosen in the presented results.

-- We added this info into the corresponding section.

2. For completeness it would be ideal to also include a test of the convergence of the temporal error by xing a mesh size and studying the error as $t->0$.

-- We added a time convergence table.

3. Last, the analysis of the fully discrete problem is not provided. At the very least the authors should provide a discussion that goes a bit further than merely stating that extension to the high order case is leasy".

-- We added a remark about this point with references to such semi-discrete to fully discrete analysis. 


\title{
Analysis of a mixed discontinuous Galerkin method for instationary Darcy flow
}

\author{
Vadym Aizinger ${ }^{\star}$ Andreas Rupp • \\ Jochen Schütz • Peter Knabner
}

Received: date / Accepted: date

\begin{abstract}
We present an a priori stability and convergence analysis of a new mixed discontinuous Galerkin scheme applied to the instationary Darcy problem. The analysis accounts for a spatially and temporally varying permeability tensor in all estimates. The proposed method is stabilized using penalty terms in the primary and the flux unknowns.
\end{abstract}

Keywords mixed discontinuous Galerkin method · local discontinuous Galerkin method · instationary Darcy problem · stability and error analysis

\section{Introduction}

The discontinuous Galerkin (DG) method first introduced in [24] for a transport equation has become one of the most widely used numerical schemes in many areas of CFD. Particularly important for a wide adoption of this method was the appearance of discretization techniques for second order terms such as the Laplace operator. Those techniques currently include:

- discretizations operating directly on the scalar PDE similarly to the classical finite element method that originate from the interior penalty (IP) schemes introduced in the late 1970s and early 1980s for elliptic and parabolic equations (cf. [4] for an overview);

* Corresponding author.

V. Aizinger · A. Rupp · P. Knabner

Chair of Applied Mathematics 1, University of Erlangen-Nürnberg, Cauerstraße 11, 91058 Erlangen, Germany

E-mail: \{aizinger,rupp,knabner\}@math.fau.de

J. Schütz

Faculty of Sciences, Hasselt University, Campus Diepenbeek, Agoralaan Gebouw D, 3590

Diepenbeek, Belgium

E-mail: jochen.schuetz@uhasselt.be 
- DG methods based on the mixed formulation, in particular the local discontinuous Galerkin (LDG) method first introduced by Cockburn and Shu for convection-diffusion systems in [13];

- DG discretizations using staggered approach for flux reconstruction [18].

The mixed DG formulations - also considered in the present work - replace each second (or higher) order equation with a first order system by introducing auxiliary flux variable(s). As opposed to methods from the IP family, mixed DG schemes also work for piecewise constant approximation spaces, the latter being exactly equivalent to the cell-centered finite volume method. In addition, this type of DG discretization is the foundation of the compact discontinuous Galerkin (CDG) [22] and the hybridized discontinuous Galerkin (HDG) [7] methods. For an overview of the current state of development of DG methods and of DG formulations for various types of applications, we refer the interested reader to a number of excellent review articles $[10,30,32,21,25,26]$.

The original work by Cockburn and Shu [13] on the LDG method for the convection-diffusion equation with constant coefficients considers a symmetrized mixed formulation distinctly differing from our approach. A corresponding elliptic problem is analyzed in great detail in [8] where some sharp convergence results for the primary and the flux unknowns are presented for the first time. The analysis in [8] considers the Poisson equation, and the estimates for the primary and the flux unknowns are conducted separately producing convergence rates of $\mathcal{O}\left(h^{k+1}\right)$ and $\mathcal{O}\left(h^{k}\right)$ in the $L^{2}$-norm, respectively for equal-order approximations of order $k$ for both unknowns. A Fourier-type analysis (performed by re-casting one-dimensional DG discretizations as finite differences stencils) is demonstrated by Zhang and Shu in [31]. Dawson in [14] presents an LDG scheme (that formulation also includes some reconstruction ideas) based on approximation spaces one order higher for the flux variables proving convergence rates of $\mathcal{O}\left(h^{k+1}\right)$ for all unknowns. Similar LDG methods are also investigated in $[9,3]$.

Analysis of numerical methods for Darcy flow usually considers a somewhat more general setting than a classical (linear) diffusion equation or Poisson problem. The important issues for this specific application include spatially and (possibly also temporally) varying tensor-valued coefficients; the studies also should ideally evaluate the behavior of the numerical method in cases of discontinuous permeability. An intermediate step between a constant diffusion and the full Darcy problem in the case of the LDG method was covered by the work of Cockburn and Dawson [12] where a space/time variable non-negative diffusion coefficient was analyzed for the first time. Methods for Darcy's flow have been formulated and analyzed in the well-known paper by Brezzi et al. [6] that also discusses the well-posedness of the proposed DG formulation; the scheme presented there is stabilized by a residual-based approach based on ideas in [20] and [17] and works for polynomial orders $k \geq 1$. Other discontinuous Galerkin methods for Darcy flow are examined in [27], a more general problem than considered in the present work is analyzed in [28]. Similar ideas as in [6] augmented by a posteriori error estimates are investigated in [5]. 
Somewhat closer to the present study is the work of Perugia and Schötzau [23] where an $h p$-error analysis of an LDG method for stationary diffusion problem with variable coefficients is carried out. Their analysis relies on test functions similar to ours; however the non-symmetric formulation of the second-order term is dealt with by introducing a third auxiliary unknown (reminiscent of our work in [3]) instead of the direct approach used by us, also the stabilization terms of order $\mathcal{O}\left(h^{-1}\right)$ similar to [8] are employed rather than stabilization terms of orders $\mathcal{O}(h)$ and $\mathcal{O}(1)$ utilized in our formulation.

The DG scheme presented in this work is stabilized using penalties in both, the primary and the flux unknowns (the latter also account for variable coefficients) and thus fits into the unified DG framework established in [4]. The particular goals of the present study are twofold: First, we attempt to create a scheme with smaller stabilization terms in hope to reduce the numerical diffusion. Second, we extend the analysis techniques utilized in $[1,2]$ for nonlinear two- and three-dimensional shallow water equations to Darcy's system. The strengths of our approach include using the 'traditional' type of DG analysis and a 'natural' DG norm to simultaneously obtain convergence results for both, the primary and the flux unknowns. All estimates incorporate variable diffusion/permeability coefficients and inhomogeneous Dirichlet and Neumann boundary conditions.

The paper is structured as follows. In the following section, we state the investigated problem and give its semi-discrete DG formulation. The main results are presented in Section 3; those include the stability and the error estimates of the proposed DG scheme. In Section 4, some numerical studies are conducted. A short conclusion and outlook section wraps up this paper.

\section{Problem formulation}

\section{Mathematical model}

We consider the following instationary Darcy problem on a polyhedral (polygonal) domain $\Omega \subset \mathbb{R}^{d}$ with $\partial \Omega=\Gamma_{\mathrm{D}} \cup \Gamma_{\mathrm{N}}$ and compact closure

$$
\left\{\begin{aligned}
\partial_{t} u+\nabla \cdot(K(t, \mathbf{x}) \mathbf{q}) & =f(t, \mathbf{x}) & & \text { in }(0, T) \times \Omega, \\
\mathbf{q}+\nabla u & =0 & & \text { in }(0, T) \times \Omega, \\
u(0, \mathbf{x}) & =u_{0}(\mathbf{x}) & & \text { in } \Omega \\
u & =u_{D}(t, \mathbf{x}) & & \text { on }(0, T) \times \Gamma_{\mathrm{D}}, \\
K(t, \mathbf{x}) \mathbf{q} \cdot \boldsymbol{\nu} & =g_{\mathrm{N}}(t, \mathbf{x}) & & \text { on }(0, T) \times \Gamma_{\mathrm{N}}
\end{aligned}\right.
$$

for given $f \in L^{2}\left(0, T ; L^{2}(\Omega)\right), K \in L^{\infty}\left(0, T ; W^{1, \infty}(\Omega)^{d, d}\right)$ uniformly symmetric positive definite, and $u_{\mathrm{D}}, g_{\mathrm{N}} \in L^{2}\left(0, T ; H^{1}(\Omega)\right)$. Here, $\boldsymbol{\nu}$ denotes the outward unit normal to $\partial \Omega$.

Remark 1. i) Uniform symmetric positive definiteness of $K$ implies the existence of constants $C_{K}, c_{K}>0$ independent of $t \in(0, T)$ and $\mathbf{x} \in \Omega$ such that for almost every (a.e.) $t \in(0, T), \mathbf{x} \in \Omega$

$$
c_{K}\|\xi\|_{2}^{2} \leq \xi \cdot K(t, \mathbf{x}) \xi \leq C_{K}\|\xi\|_{2}^{2}, \quad \forall \xi \in \mathbb{R}^{d} .
$$


In addition, we require $C_{K} \geq\|K(t)\|_{L^{\infty}(\Omega)}$ for a.e. $t \in(0, T)$.

ii) $K \in W^{1, \infty}(\Omega)^{d, d}$ (in space) implies Lipschitz continuity on $\Omega$.

iii) Assuming that $u \in H^{1}\left(0, T ; L^{2}(\Omega)\right) \cap L^{2}\left(0, T ; H^{2}(\Omega)\right)$ (the minimum regularity needed for our convergence analysis) we have $\mathbf{q} \in L^{2}\left(0, T ; H^{1}(\Omega)^{d}\right)$ implies that $K \mathbf{q} \in L^{2}\left(0, T ; H^{1}(\Omega)^{d}\right)$ and that the trace of $K \mathbf{q}$ on $\Gamma_{N}$ (or, for that matter, on any Lipschitz submanifold $\Gamma \subset \bar{\Omega})$ is well defined.

\section{Weak problem}

Here, we define test functions $\varphi_{u} \in H^{1}\left(0, T ; C^{\infty}(\bar{\Omega})\right)$ (scalar) and $\varphi_{\mathbf{q}} \in$ $L^{2}\left(0, T ; C^{\infty}(\bar{\Omega})^{d}\right)$ (vector), multiply (1) by these test functions, and integrate by parts. Thus we get for a.e. $s \in(0, T)$ and any Lipschitz domain $\Omega_{e} \subset \Omega$

$$
\begin{aligned}
& \int_{0}^{s} \int_{\Omega_{e}}\left(\partial_{t} u\right) \varphi_{u} d \mathbf{x} d t-\int_{0}^{s} \int_{\Omega_{e}}(K \mathbf{q}) \cdot \nabla \varphi_{u} d \mathbf{x} d t+\int_{0}^{s} \int_{\partial \Omega_{e} \backslash \Gamma_{\mathrm{N}}} K \mathbf{\nu} \cdot \boldsymbol{\nu} \varphi_{u} d \sigma d t \\
& \quad+\int_{0}^{s} \int_{\partial \Omega_{e} \cap \Gamma_{\mathrm{N}}} g_{\mathrm{N}} \varphi_{u} d \sigma d t=\int_{0}^{s} \int_{\Omega_{e}} f \varphi_{u} d \mathbf{x} d t \\
& \int_{0}^{s} \int_{\Omega_{e}} \mathbf{q} \cdot \varphi_{\mathbf{q}} d \mathbf{x} d t-\int_{0}^{s} \int_{\Omega_{e}} u\left(\nabla \cdot \varphi_{\mathbf{q}}\right) d \mathbf{x} d t+\int_{0}^{s} \int_{\partial \Omega_{e} \cap \Gamma_{\mathrm{D}}} u_{\mathrm{D}} \boldsymbol{\varphi}_{\mathbf{q}} \cdot \boldsymbol{\nu} d \sigma d t \\
& \quad+\int_{0}^{s} \int_{\partial \Omega_{e} \backslash \Gamma_{\mathrm{D}}} u \boldsymbol{\varphi}_{\mathbf{q}} \cdot \boldsymbol{\nu} d \sigma d t=0, \\
& \int_{\Omega_{e}} u(0, \mathbf{x}) \varphi_{u}(0, x) d \mathbf{x}=\int_{\Omega_{e}} u_{0}(x) \varphi_{u}(0, \mathbf{x}) d \mathbf{x} .
\end{aligned}
$$

\section{DG formulation}

In the following, $\mathcal{T}_{h}=\left\{\mathcal{K}_{i}: i=1, \ldots, N_{\mathrm{el}}\right\}$ denotes a $d$-dimensional nonoverlapping polyhedral (polygonal) partition of $\Omega$ (see [15, Def. 1.12]) that is assumed to be geometrically conformal in the sense of [16, Def. 1.55]. This condition implies that for any two mesh elements that have a non-empty $d-$ 1-dimensional intersection, this intersection is a face of both elements; this condition is purely technical and serves to simplify the treatment of traces and boundary integral terms; all results can be easily extended to non-conformal meshes. We denote by $\mathcal{F}=\mathcal{F}\left(\mathcal{T}_{h}\right)$ the set of faces, by $\mathcal{F}_{\mathrm{I}}$ the set of interior faces, and by $\mathcal{F}_{\mathrm{E}}$ the set of exterior faces. We further assume that $\mathcal{F}_{\mathrm{E}}=$ $\mathcal{F}_{\mathrm{D}} \cup \mathcal{F}_{\mathrm{N}}$, where $\mathcal{F}_{\mathrm{D}}\left(\mathcal{F}_{\mathrm{N}}\right)$ is the set of Dirichlet (Neumann) boundary faces and write $h_{F}=\operatorname{diam} F$ for the diameter of $F \in \mathcal{F}$. The test- and ansatz-spaces for our DG method are defined as the broken polynomial spaces of order $k$

$\mathbb{P}_{k}^{d}\left(\mathcal{T}_{h}\right):=\left\{\mathbf{v} \in L^{2}(\Omega)^{d}: \mathbf{v}_{\mid \mathcal{K}, \mathcal{K} \in \mathcal{T}_{h}}\right.$ is a polynomial of total degree at most $\left.k\right\}$.

We denote the $L^{2}$-projection by $\pi: L^{2}(\mathcal{K})^{d} \rightarrow \mathbb{P}_{k}^{d}(\mathcal{K})$ and recognize that it is a well-defined, linear, orthogonal (with respect to the standard $L^{2}$-scalar product) projection with $\|\boldsymbol{\pi}\|=1$. The $L^{2}$-projection-error is denoted by

$$
\theta_{\mathbf{v}}:=\boldsymbol{\pi}(\mathbf{v})-\mathbf{v}, \quad \forall \mathbf{v} \in L^{2}(\mathcal{K})^{d} .
$$


For a scalar function $w$ and a vector function $\mathbf{v}$ defined on $\Omega$ and smooth enough to posses a unique trace on $\partial \mathcal{K}, \forall \mathcal{K} \in \mathcal{T}_{h}$, we define the average $\{[\cdot]\}$ and the jump $\llbracket \cdot \rrbracket$ on $\partial \mathcal{K}_{i} \cap \partial \mathcal{K}_{j}$ for neighboring mesh elements $\mathcal{K}_{i}, \mathcal{K}_{j} \in \mathcal{T}_{h}, \mathcal{K}_{i} \neq$ $\mathcal{K}_{j}, \mathcal{K}_{i} \cap \mathcal{K}_{j} \neq \emptyset$ in the following standard way:

$$
\begin{aligned}
& \{[w]\}=\frac{1}{2}\left(w_{\mid \mathcal{K}_{i}}+w_{\mid \mathcal{K}_{j}}\right), \quad \llbracket w \rrbracket=w_{\mid \mathcal{K}_{i}} \boldsymbol{\nu}_{\mathcal{K}_{i}}+w_{\mid \mathcal{K}_{j}} \boldsymbol{\nu}_{\mathcal{K}_{j}}, \\
& \{[\mathbf{v}]\}=\frac{1}{2}\left(\mathbf{v}_{\mid \mathcal{K}_{i}}+\mathbf{v}_{\mid \mathcal{K}_{j}}\right), \quad \quad \llbracket \mathbf{v} \rrbracket=\mathbf{v}_{\mid \mathcal{K}_{i}} \cdot \boldsymbol{\nu}_{\mathcal{K}_{i}}+\mathbf{v}_{\mid \mathcal{K}_{j}} \cdot \boldsymbol{\nu}_{\mathcal{K}_{j}},
\end{aligned}
$$

where $\boldsymbol{\nu}_{\mathcal{K}}$ is defined as the outward unit normal with respect to $\mathcal{K}$ (we drop element subscripts for normals in the following). Note, that a jump in a scalar variable is a vector, whereas a jump of a vector is a scalar. In this notation, the well-known product rule for jumps reads:

$$
\llbracket w \mathbf{v} \rrbracket=\{[w]\} \llbracket \mathbf{v} \rrbracket+\llbracket w \rrbracket \cdot\{[\mathbf{v}]\} .
$$

Using the above definitions we can formulate the semi-discrete

Finite Element Problem. For almost every $s \in(0, T)$ seek $\left(u_{h}(s), \mathbf{q}_{h}(s)\right) \in$ $\mathbb{P}_{k}^{1}\left(\mathcal{T}_{h}\right) \times \mathbb{P}_{k}^{d}\left(\mathcal{T}_{h}\right)$ such that the following holds $\forall \mathcal{K} \in \mathcal{T}_{h}, \forall\left(\varphi_{u}, \varphi_{\mathbf{q}}\right) \in \mathbb{P}_{k}^{1}\left(\mathcal{T}_{h}\right) \times$ $\mathbb{P}_{k}^{d}\left(\mathcal{T}_{h}\right)$ :

$$
\begin{aligned}
& \int_{\mathcal{K}}\left(\partial_{t} u_{h}\right) \varphi_{u} d \mathbf{x}-\int_{\mathcal{K}} \boldsymbol{\pi}\left(K \mathbf{q}_{h}\right) \cdot \nabla \varphi_{u} d \mathbf{x}+\int_{\partial \mathcal{K}} \widehat{K \mathbf{q}_{h}} \varphi_{u} d \sigma=\int_{\mathcal{K}} f \varphi_{u} d \mathbf{x}, \\
& \int_{\mathcal{K}} \mathbf{q}_{h} \cdot \varphi_{\mathbf{q}} d \mathbf{x}-\int_{\mathcal{K}} u_{h}\left(\nabla \cdot \varphi_{\mathbf{q}}\right) d \mathbf{x}+\int_{\partial \mathcal{K}} \widehat{u}_{h} \boldsymbol{\varphi}_{\mathbf{q}} \cdot \boldsymbol{\nu} d \sigma=0, \\
& \int_{\mathcal{K}} u_{h}(0) \varphi_{u}(0) d \mathbf{x}=\int_{\mathcal{K}} u_{0} \varphi_{u}(0) d \mathbf{x}, \quad \text { for } s=0,
\end{aligned}
$$

where $\eta, \mu>0$ are the penalty coefficients and $\widehat{K \mathbf{q}_{h}}, \widehat{u}_{h}$ are the boundary fluxes defined as

$$
\begin{aligned}
\widehat{K \mathbf{q}_{h}} & = \begin{cases}\boldsymbol{\pi}\left(K \mathbf{q}_{h}\right) \cdot \boldsymbol{\nu}+\eta h_{F}\left(u_{h}-u_{D}\right), & \text { on } \Gamma_{D}, \\
g_{N}, & \text { on } \Gamma_{N}, \\
\left\{\left[\boldsymbol{\pi}\left(K \mathbf{q}_{h}\right)\right]\right\} \cdot \boldsymbol{\nu}+\eta h_{F} \llbracket u_{h} \rrbracket \cdot \boldsymbol{\nu}, & \text { otherwise, }\end{cases} \\
\widehat{u}_{h} & = \begin{cases}u_{D}, & \text { on } \Gamma_{D} \\
u_{h}+\mu\left(\boldsymbol{\pi}\left(K \mathbf{q}_{h}\right) \cdot \boldsymbol{\nu}-g_{N}\right), & \text { on } \Gamma_{N}, \\
\left\{\left[u_{h}\right]\right\}+\mu \llbracket \boldsymbol{\pi}\left(K \mathbf{q}_{h}\right) \rrbracket, & \text { otherwise. }\end{cases}
\end{aligned}
$$

Remark 2. Substituting the solution of (2.a) - (2.c) into Finite Element Problem produces a consistency error since $\boldsymbol{\pi}(K \mathbf{q})$ is generally not singlevalued on inter-element boundaries. Replacing $\boldsymbol{\pi}(K \mathbf{q})$ with Kq restores the full consistency.

Remark 3. The following analysis treats the semi-discrete (method of lines) formulation of our method. The reasons for this choice are twofold:

- simplifying the analysis technique and 
- focusing on the space discretization since it is the main contribution of the present work.

Extending our work to a fully discrete analysis can be performed along the lines of [19, Th. 7.29 - 7.31] for any $\theta$-scheme with $\theta \geq 0.5$ (the trapezoidal method in our simulations has $\theta=0.5$ ) under assumption of sufficient regularity of the solution in time. More time stepping algorithms are considered in [29], where Chapters 8 and 10 introduce rather general techniques that can be also used in our context to obtain the convergence orders of the fully discrete scheme.

\section{Stability and error analysis}

For the analysis of the DG method, we need a special definition of regularity of $\left(\mathcal{T}_{h}\right)_{h>0}$ (similar to the definitions given in [15, Def. 1.38]).

Definition 1 (Shape and Contact Regularity - $\underline{\mathrm{SCR}})$. A family of meshes $\left(\mathcal{T}_{h}\right)_{h>0}$ is said to be shape and contact regular (short regular) if there exist constants $\lambda_{1}, \lambda_{2}, \lambda_{3}$ independent of $h$, and if each $\mathcal{T}_{h}$ admits a geometrically conformal, matching simplicial submesh ([15, Def. 1.37]) $\widetilde{\mathcal{T}}_{h}$ such that

i) $\lambda_{1} h_{\widetilde{\mathcal{K}}} \leq \rho_{\widetilde{\mathcal{K}}}, \forall \widetilde{\mathcal{K}} \in \widetilde{\mathcal{T}}_{h}$, where $\rho_{\widetilde{\mathcal{K}}}$ is the diameter of the largest ball that can be inscribed in $\widetilde{\mathcal{K}}$.

ii) $\lambda_{2} h_{\mathcal{K}} \leq h_{\widetilde{\mathcal{K}}}, \forall \widetilde{\mathcal{K}} \in \widetilde{\mathcal{T}}_{h}$ with $\widetilde{\mathcal{K}} \subset \mathcal{K}$,

iii) $\lambda_{3} h \leq h_{F}, \forall F \in \mathcal{F}$.

Remark 4. Our analysis extensively utilizes usual tools such as Young's inequality and the Bramble-Hilbert Lemma. Other standard results from the theory of finite elements enter in a form customized to the particular proof techniques used in this paper.

Lemma 1 (Discrete Trace Inequality - DTI). For $\left(\mathcal{T}_{h}\right)_{h>0}$ a regular mesh family as in Definition 1, for all $h>0$, and all $\mathbf{p} \in \mathbb{P}_{k}^{d}\left(\mathcal{T}_{h}\right)$, we have

$$
h^{1 / 2} \sum_{F \in \mathcal{F}}\|\mathbf{p}\|_{L^{2}(F)^{d}} \leq C_{t r} \sum_{\mathcal{K} \in \mathcal{T}_{h}}\|\mathbf{p}\|_{L^{2}(\mathcal{K})^{d}}=C_{t r}\|\mathbf{p}\|_{L^{2}(\Omega)^{d}},
$$

where $C_{\text {tr }}$ only depends on $\lambda_{i}, i=1,2,3 ; d$, and $k$. For $F$ shared by elements $\mathcal{K}_{i}$ and $\mathcal{K}_{j}$, the first term above is considered to contain both traces:

$$
\|\mathbf{p}\|_{L^{2}(F)}=\left\|\mathbf{p}_{\mid \mathcal{K}_{i}}\right\|_{L^{2}(F)}+\left\|\mathbf{p}_{\mid \mathcal{K}_{j}}\right\|_{L^{2}(F)} .
$$

Proof. This result follows directly from [15, Lemma 1.46] and Definition 1.

Lemma 2 (Projection Trace Inequality - $\underline{\mathrm{PTI}})$. Let $\left(\mathcal{T}_{h}\right)_{h>0}$ be a regular mesh sequence with parameters $\lambda_{i}, i=1,2,3$. Then there exists a constant $C_{k}$ that only depends on $\lambda_{i}, i=1,2,3 ; d$, and $k$ such that for $k=0,1,2, \ldots$ and for $\mathbf{q}:=-\nabla u$
i) $\sum_{F \in \mathcal{F}}\left\|\theta_{u}\right\|_{L^{2}(F)} \leq C_{k} \sum_{\mathcal{K} \in \mathcal{T}_{h}} h_{\mathcal{K}}^{k+1 / 2}|u|_{H^{k+1}(\mathcal{K})}$
$\forall u \in H^{k+1}(\Omega)$ 
ii) $\sum_{F \in \mathcal{F}}\left\|\theta_{\mathbf{q}}\right\|_{L^{2}(F)^{d}} \leq C_{k} \sum_{\mathcal{K} \in \mathcal{T}_{h}} h_{\mathcal{K}}^{k+1 / 2}|u|_{H^{k+2}(\mathcal{K})}, \quad \forall u \in H^{k+2}(\Omega)$

with $\theta$ defined as in (3). The above results can be easily generalized for boundary integral terms involving jumps and averages.

Proof. The proof follows from applying the Bramble-Hilbert Lemma (see [11]) to a simplicial submesh $\widetilde{\mathcal{T}}_{h}$ of a regular mesh in the sense of Definition 1 with $\Psi=\gamma_{0} \circ(\boldsymbol{\pi}-\mathrm{id})\left(\right.$ where $\gamma_{0}$ denotes the standard trace operator on elements of $\left.\widetilde{\mathcal{T}}_{h}\right)$ and then applying [15, Lemma 1.46]. Note that $\Psi: H^{k+1}(\mathcal{K}) \rightarrow L^{2}(F)$ is continuous as a composition of continuous mappings.

For analysis purposes, we integrate the second equation in Finite Element Problem by parts, sum both equations over all elements in $\mathcal{T}_{h}$, and add the results. Compactly, this can be written as

$$
\mathcal{A}\left(u_{h}, \mathbf{q}_{h} ; \varphi_{u}, \varphi_{\mathbf{q}}\right)=\mathcal{B}\left(\varphi_{u}, \varphi_{\mathbf{q}}\right),
$$

where (after some algebraic simplifications involving properties of jumps)

$$
\begin{aligned}
& \mathcal{A}\left(u_{h}, \mathbf{q}_{h} ; \varphi_{u}, \boldsymbol{\varphi}_{\mathbf{q}}\right):=\int_{\Omega}\left(\partial_{t} u_{h}\right) \varphi_{u} d \mathbf{x}-\sum_{\mathcal{K} \in \mathcal{T}_{h}} \int_{\mathcal{K}} \boldsymbol{\pi}\left(K \mathbf{q}_{h}\right) \cdot \nabla \varphi_{u} d \mathbf{x} \\
& +\sum_{F \in \mathcal{F}_{\mathrm{I}}} \int_{F}\left\{\left[\boldsymbol{\pi}\left(K \mathbf{q}_{h}\right) \rrbracket\right\} \llbracket \llbracket \varphi_{u} \rrbracket d \sigma+\sum_{F \in \mathcal{F}_{\mathrm{D}}} \int_{F} \boldsymbol{\pi}\left(K \mathbf{q}_{h}\right) \cdot \boldsymbol{\nu} \varphi_{u} d \sigma\right. \\
& +\eta \sum_{F \in \mathcal{F}_{\mathrm{I}}} h_{F} \int_{F} \llbracket u_{h} \rrbracket \cdot \llbracket \varphi_{u} \rrbracket d \sigma+\eta \sum_{F \in \mathcal{F}_{\mathrm{D}}} h_{F} \int_{F} u_{h} \varphi_{u} d \sigma+\int_{\Omega} \mathbf{q}_{h} \cdot \boldsymbol{\varphi}_{\mathbf{q}} d \mathbf{x} \\
& \quad+\sum_{\mathcal{K} \in \mathcal{T}_{h}} \int_{\mathcal{K}} \nabla u_{h} \cdot \boldsymbol{\varphi}_{\mathbf{q}} d \mathbf{x}-\sum_{F \in \mathcal{F}_{\mathrm{I}}} \int_{F} \llbracket u_{h} \rrbracket \cdot\left\{\boldsymbol{\varphi}_{\mathbf{q}} \rrbracket d \sigma-\sum_{F \in \mathcal{F}_{\mathrm{D}}} \int_{F} u_{h} \boldsymbol{\varphi}_{\mathbf{q}} \cdot \boldsymbol{\nu} d \sigma\right. \\
& \quad+\mu \sum_{F \in \mathcal{F}_{\mathrm{I}}} \int_{F} \llbracket \boldsymbol{\pi}\left(K \mathbf{q}_{h}\right) \rrbracket \llbracket \boldsymbol{\varphi}_{\mathbf{q}} \rrbracket d \sigma+\mu \sum_{F \in \mathcal{F}_{\mathrm{N}}} \int_{F} \boldsymbol{\pi}\left(K \mathbf{q}_{h}\right) \cdot \boldsymbol{\nu} \boldsymbol{\varphi}_{\mathbf{q}} \cdot \boldsymbol{\nu} d \sigma, \\
& \mathcal{B}\left(\varphi_{u}, \varphi_{\mathbf{q}}\right):=\int_{\Omega} f \varphi_{u} d \mathbf{x}+\eta \sum_{F \in \mathcal{F}_{\mathrm{D}}} h_{F} \int_{F} u_{\mathrm{D}} \varphi_{u} d \sigma-\sum_{F \in \mathcal{F}_{\mathrm{N}}} \int_{F} g_{\mathrm{N}} \varphi_{u} d \sigma \\
& \quad-\sum_{F \in \mathcal{F}_{\mathrm{D}}} \int_{F} u_{\mathrm{D}} \boldsymbol{\varphi}_{\mathbf{q}} \cdot \boldsymbol{\nu} d \sigma+\mu \sum_{F \in \mathcal{F}_{\mathrm{N}}} \int_{F} g_{\mathrm{N}} \boldsymbol{\varphi}_{\mathbf{q}} \cdot \boldsymbol{\nu} d \sigma .
\end{aligned}
$$

We also introduce a weighted norm on partition $\mathcal{T}_{h}$ in which the stability and convergence order results will be proved:

$$
\begin{gathered}
\left\|\left(u_{h}, \mathbf{q}_{h}\right)\right\|^{2}:=\int_{\Omega} u_{h}^{2}(s, \mathbf{x}) d \mathbf{x}+\int_{0}^{s}\left[\left\|\sqrt{K} \mathbf{q}_{h}\right\|_{L^{2}(\Omega)^{d}}^{2}+\eta \sum_{F \in \mathcal{F}_{\mathrm{I}}} h_{F}\left\|\llbracket u_{h} \rrbracket\right\|_{L^{2}(F)^{d}}^{2}\right. \\
\left.+\eta \sum_{F \in \mathcal{F}_{\mathrm{D}}} h_{F}\left\|u_{h}\right\|_{L^{2}(F)}^{2}+\mu \sum_{F \in \mathcal{F}_{\mathrm{I}}}\left\|\llbracket \boldsymbol{\pi}\left(K \mathbf{q}_{h}\right) \rrbracket\right\|_{L^{2}(F)}^{2}+\mu \sum_{F \in \mathcal{F}_{\mathrm{N}}}\left\|\boldsymbol{\pi}\left(K \mathbf{q}_{h}\right)\right\|_{L^{2}(F)^{d}}^{2}\right] d t
\end{gathered}
$$


Theorem 1 (Stability of the semi-discrete problem) For $\eta>0, \mu>0$, the following statement of stability holds:

$$
\begin{aligned}
& \left\|\left(u_{h}, \mathbf{q}_{h}\right)(\cdot, s)\right\|^{2} \leq \exp (s)\left(\left\|u_{0}\right\|_{L^{2}(\Omega)}^{2}\right. \\
& \left.\quad+\int_{0}^{s}\left[C_{1}\|f\|_{L^{2}(\Omega)}^{2}+\left(C_{2} h+C_{3} h^{-1}\right)\left\|u_{D}\right\|_{L^{2}\left(\Gamma_{D}\right)}^{2}+\left(C_{4}+C_{5} h^{-1}\right)\left\|u_{N}\right\|_{L^{2}\left(\Gamma_{N}\right)}^{2}\right] d t\right)
\end{aligned}
$$

for a. e. $s \in(0, T)$ and constants $C_{1}, \ldots, C_{5}$ independent of $h$ and $s$.

Proof. Some ideas of the proof are taken from [1,2]. Testing (4) with $\varphi_{u}=$ $u_{h}, \varphi_{\mathbf{q}}=\boldsymbol{\pi}\left(K \mathbf{q}_{h}\right)$ gives us after simple algebraic manipulations

$$
\begin{aligned}
& \int_{\Omega}\left(\partial_{t} u_{h}\right) u_{h} d \mathbf{x}+\int_{\Omega} \mathbf{q}_{h} \cdot K \mathbf{q}_{h} d \mathbf{x}+\eta \sum_{F \in \mathcal{F}_{\mathrm{I}}} h_{F}\left\|\llbracket u_{h} \rrbracket\right\|_{L^{2}(F)^{d}}^{2} \\
& +\eta \sum_{F \in \mathcal{F}_{\mathrm{D}}} h_{F}\left\|u_{h}\right\|_{L^{2}(F)}^{2}+\mu \sum_{F \in \mathcal{F}_{\mathrm{I}}}\left\|\llbracket \boldsymbol{\pi}\left(K \mathbf{q}_{h}\right) \rrbracket\right\|_{L^{2}(F)}^{2}+\mu \sum_{F \in \mathcal{F}_{\mathrm{N}}}\left\|\boldsymbol{\pi}\left(K \mathbf{q}_{h}\right)\right\|_{L^{2}(F)^{d}}^{2} \\
& =\underbrace{\int_{\Omega} f u_{h} d \mathbf{x}}_{=: \Xi_{1}}+\underbrace{\eta \sum_{F \in \mathcal{F}_{\mathrm{D}}} h_{F} \int_{F} u_{\mathrm{D}} u_{h} d \sigma}_{=: \Xi_{2}}-\underbrace{\sum_{F \in \mathcal{F}_{N}} \int_{F} g_{\mathrm{N}} u_{h} d \sigma}_{=: \Xi_{3}} \\
& -\underbrace{\sum_{F \in \mathcal{F}_{\mathrm{D}}} \int_{F} u_{\mathrm{D}} \boldsymbol{\pi}\left(K \mathbf{q}_{h}\right) \cdot \boldsymbol{\nu} d \sigma}_{=: \Xi_{4}}+\underbrace{\mu \sum_{F \in \mathcal{F}_{\mathrm{N}}} \int_{F} g_{\mathrm{N}} \boldsymbol{\pi}\left(K \mathbf{q}_{h}\right) \cdot \boldsymbol{\nu} d \sigma}_{=: \Xi_{5}}
\end{aligned}
$$

Estimating $\Xi_{1}, \ldots, \Xi_{5}$ we obtain:

$$
\begin{aligned}
\left|\Xi_{1}\right| & \leq\|f\|_{L^{2}(\Omega)}\left\|u_{h}\right\|_{L^{2}(\Omega)} \leq \frac{1}{4}\left\|u_{h}\right\|_{L^{2}(\Omega)}^{2}+\frac{C_{1}}{2}\|f\|_{L^{2}(\Omega)}^{2}, \\
\left|\Xi_{2}\right| & \leq \eta \sum_{F \in \mathcal{F}_{\mathrm{D}}} h_{F} \int_{F}\left(\frac{1}{4} u_{h}^{2}+u_{\mathrm{D}}^{2}\right) d \mathbf{x} \leq \frac{\eta}{4} \sum_{F \in \mathcal{F}_{\mathrm{D}}} h_{F}\left\|u_{h}^{2}\right\|_{L^{2}(F)}^{2}+\frac{C_{2} h}{2}\left\|u_{\mathrm{D}}\right\|_{L^{2}\left(\Gamma_{\mathrm{D}}\right)}^{2}, \\
\left|\Xi_{3}\right| & \leq \sum_{F \in \mathcal{F}_{\mathrm{N}}}\left(\frac{h_{F}}{4 C_{\mathrm{tr}}^{2}}\left\|u_{h}\right\|_{L^{2}(F)}^{2}+\frac{C_{\mathrm{tr}}^{2}}{h_{F}}\left\|g_{\mathrm{N}}\right\|_{L^{2}(F)}^{2}\right) \leq \underbrace{\frac{1}{4}\left\|u_{h}\right\|_{L^{2}(\Omega)}^{2}}_{[\underline{\mathbf{D T I}}]}+\underbrace{\frac{C_{5}}{2 h}\left\|g_{\mathrm{N}}\right\|_{L^{2}\left(\Gamma_{\mathrm{N}}\right)}^{2}}_{[\underline{\mathbf{S C R}]}}, \\
\left|\Xi_{4}\right| & \leq \underbrace{}_{F \in \mathcal{F}_{\mathrm{D}}}\left(\frac{1}{2} \frac{h_{F}}{C_{\mathrm{tr}}^{2} C_{K}}\left\|\boldsymbol{\pi}\left(K \mathbf{q}_{h}\right)\right\|_{L^{2}(F)}^{2}+\frac{1}{2} \frac{C_{\mathrm{tr}}^{2} C_{K}}{h_{F}}\left\|u_{\mathrm{D}}\right\|_{L^{2}(F)}^{2}\right) \\
& \leq \underbrace{\frac{1}{2}\left\|\sqrt{K} \mathbf{q}_{h}\right\|_{L^{2}(\Omega)^{d}}^{2}}_{[\operatorname{Rem} .1, \underline{\mathbf{D T I}}]}+\frac{C_{3}}{2 h}\left\|u_{\mathrm{D}}\right\|_{L^{2}\left(\Gamma_{\mathrm{D}}\right)}^{2}, \\
\left|\Xi_{5}\right| & \leq \frac{\mu}{2} \sum_{F \in \mathcal{F}_{\mathrm{N}}}\left\|\boldsymbol{\pi}\left(K \mathbf{q}_{h}\right)\right\|_{L^{2}(F)^{d}}^{2}+\frac{C_{4}}{2}\left\|g_{\mathrm{N}}\right\|_{L^{2}\left(\Gamma_{\mathrm{N}}\right)}^{2} .
\end{aligned}
$$

Integrating over $(0, s)$ and using Grönwall's inequality completes the proof. 
To analyze the convergence of our semi-discrete scheme we need to define some new operators. In order to do so, let us assume that $(u, \mathbf{q})$ is the solution of (2.a) - (2.c) and $\left(u_{h}, \mathbf{q}_{h}\right)$ is the solution of Finite Element Problem. Then we define

$$
\begin{array}{lll}
e_{u}:=u_{h}-\boldsymbol{\pi}(u), & \theta_{u}:=\boldsymbol{\pi}(u)-u, & \mathbb{U}_{u}:=u_{h}-u, \\
e_{\mathbf{q}}:=\mathbf{q}_{h}-\boldsymbol{\pi}(\mathbf{q}), & \theta_{\mathbf{q}}:=\boldsymbol{\pi}(\mathbf{q})-\mathbf{q}, & \mathbb{U}_{\mathbf{q}}:=\mathbf{q}_{h}-\mathbf{q}, \\
e_{K \mathbf{q}}:=\boldsymbol{\pi}\left(K \mathbf{q}_{h}\right)-\boldsymbol{\pi}(K \mathbf{q}), & \theta_{K \mathbf{q}}:=\boldsymbol{\pi}(K \mathbf{q})-K \mathbf{q}, & \mathbb{U}_{K \mathbf{q}}:=\boldsymbol{\pi}\left(K \mathbf{q}_{h}\right)-K \mathbf{q}
\end{array}
$$

Theorem 2 (Convergence order estimate) For $\mathcal{T}_{h}$ a regular family of polygonal/polyhedral partitions of $\Omega, K \in L^{\infty}\left(0, T ; W^{k+1, \infty}(\Omega)^{d, d}\right)$, and $\eta>$ $0, \mu>0$, there exists $C>0$ independent of $h$ such that

$$
\left\|\left(\mathbb{U}_{u}, \mathbb{U}_{\mathbf{q}}\right)(\cdot, s)\right\|^{2} \leq C\left(h^{2 k+2}+h^{2 k+1}+h^{2 k}\right) \quad \text { for a.e. } s \in(0, T)
$$

Proof. Some ideas of the proof are taken from [1,2]. As opposed to the stability proof in Theorem 1, the error estimate has also to account for the consistency errors in Finite Element Problem caused by the $L^{2}$-projection $\boldsymbol{\pi}(K \mathbf{q})$. Thus substituting the solution for (2.a) $-(2 . \mathrm{c})$ into $\mathcal{A}(\cdot, \cdot ; \cdot, \cdot)$ results in the following expression:

$$
\begin{aligned}
& \mathcal{A}\left(u, \mathbf{q} ; \varphi_{u}, \boldsymbol{\varphi}_{\mathbf{q}}\right)=\mathcal{B}\left(\varphi_{u}, \boldsymbol{\varphi}_{\mathbf{q}}\right)+\sum_{F \in \mathcal{F}_{\mathrm{I}}} \int_{F}(\{[\boldsymbol{\pi}(K \mathbf{q})]\}-K \mathbf{q}) \cdot \llbracket \varphi_{u} \rrbracket d \sigma \\
& \quad+\sum_{F \in \mathcal{F}_{\mathrm{D}}} \int_{F}(\boldsymbol{\pi}(K \mathbf{q})-K \mathbf{q}) \cdot \boldsymbol{\nu} \varphi_{u} d \sigma+\mu \sum_{F \in \mathcal{F}_{\mathrm{I}}} \int_{F} \llbracket \boldsymbol{\pi}(K \mathbf{q}) \rrbracket \llbracket \boldsymbol{\varphi}_{\mathbf{q}} \rrbracket d \sigma \\
& \quad+\mu \sum_{F \in \mathcal{F}_{\mathrm{N}}} \int_{F}\left(\boldsymbol{\pi}(K \mathbf{q}) \cdot \boldsymbol{\nu}-g_{\mathrm{N}}\right) \boldsymbol{\varphi}_{\mathbf{q}} \cdot \boldsymbol{\nu} d \sigma, \quad \forall\left(\varphi_{u}, \boldsymbol{\varphi}_{\mathbf{q}}\right) \in \mathbb{P}_{k}^{1}\left(\mathcal{T}_{h}\right) \times \mathbb{P}_{k}^{d}\left(\mathcal{T}_{h}\right) .
\end{aligned}
$$

Next, exploiting the linearity of forms $\mathcal{A}$ and $\mathcal{B}$ in each argument and choosing the test functions as $\varphi_{u}=e_{u}$ and $\boldsymbol{\varphi}_{\mathbf{q}}=e_{K \mathbf{q}}$ we obtain the error equation

$$
\begin{gathered}
\mathcal{A}\left(\mathbb{U}_{u}, \mathbb{U}_{\mathbf{q}} ; e_{u}, e_{K \mathbf{q}}\right)=-\sum_{F \in \mathcal{F}_{\mathrm{I}}} \int_{F}\left\{\left[\theta_{K \mathbf{q}}\right]\right\} \cdot \llbracket e_{u} \rrbracket d \sigma-\sum_{F \in \mathcal{F}_{\mathrm{D}}} \int_{F} \theta_{K \mathbf{q}} \cdot \boldsymbol{\nu} e_{u} d \sigma \\
-\mu \sum_{F \in \mathcal{F}_{\mathrm{I}}} \int_{F} \llbracket \theta_{K \mathbf{q}} \rrbracket \llbracket e_{K \mathbf{q}} \rrbracket d \sigma-\mu \sum_{F \in \mathcal{F}_{\mathrm{N}}} \int_{F} \theta_{K \mathbf{q}} \cdot \boldsymbol{\nu} e_{K \mathbf{q}} \cdot \boldsymbol{\nu} d \sigma
\end{gathered}
$$


where

$$
\begin{aligned}
& \mathcal{A}\left(\mathbb{U}_{u}, \mathbb{U}_{\mathbf{q}} ; e_{u}, e_{K \mathbf{q}}\right)=\int_{\Omega} \partial_{t}\left(e_{u}+\theta_{u}\right) e_{u} d \mathbf{x}-\sum_{\mathcal{K} \in \mathcal{T}_{h}} \int_{\mathcal{K}}\left(e_{K \mathbf{q}}+\theta_{K \mathbf{q}}\right) \cdot \nabla e_{u} d \mathbf{x} \\
& +\sum_{F \in \mathcal{F}_{\mathrm{I}}} \int_{F}\left\{\left[e_{K \mathbf{q}}\right]\right\} \cdot \llbracket e_{u} \rrbracket d \sigma+\sum_{F \in \mathcal{F}_{\mathrm{D}}} \int_{F} e_{K \mathbf{q}} \cdot \boldsymbol{\nu} e_{u} d \sigma \\
& +\eta \sum_{F \in \mathcal{F}_{\mathrm{I}}} h_{F} \int_{F}(\underbrace{\llbracket e_{u}+\theta_{u} \rrbracket}_{=\llbracket e_{u} \rrbracket+\llbracket \theta_{u} \rrbracket}) \cdot \llbracket e_{u} \rrbracket d \sigma+\eta \sum_{F \in \mathcal{F}_{\mathrm{D}}} h_{F} \int_{F}\left(e_{u}+\theta_{u}\right) e_{u} d \sigma \\
& +\int_{\Omega}\left(\mathbf{q}_{h}-\mathbf{q}\right) \cdot e_{K \mathbf{q}} d \mathbf{x}+\sum_{\mathcal{K} \in \mathcal{T}_{h}} \int_{\mathcal{K}}\left(\nabla e_{u}+\nabla \theta_{u}\right) \cdot e_{K \mathbf{q}} d \mathbf{x}-\sum_{F \in \mathcal{F}_{I}} \int_{F} \llbracket e_{u}+\theta_{u} \rrbracket \cdot\left\{\left[e_{K \mathbf{q}}\right]\right\} d \sigma \\
& -\sum_{F \in \mathcal{F}_{\mathrm{D}}} \int_{F}\left(e_{u}+\theta_{u}\right) e_{K \mathbf{q}} \cdot \boldsymbol{\nu} d \sigma+\mu \sum_{F \in \mathcal{F}_{\mathrm{I}}} \int_{F} \llbracket e_{K \mathbf{q}} \rrbracket^{2} d \sigma+\mu \sum_{F \in \mathcal{F}_{\mathrm{N}}} \int_{F}\left(e_{K \mathbf{q}} \cdot \boldsymbol{\nu}\right)^{2} d \sigma
\end{aligned}
$$

Using the following simplifications

i) $\int_{\Omega} \partial_{t} \theta_{u} e_{u} d \mathbf{x}=0$

ii) $\sum_{\mathcal{K} \in \mathcal{T}_{h}} \int_{\mathcal{K}} \theta_{K \mathbf{q}} \cdot \nabla e_{u} d \mathbf{x}=0$

iii) $\int_{\Omega}\left(\mathbf{q}_{h}-\mathbf{q}\right) \cdot e_{K \mathbf{q}} d \mathbf{x}=\int_{\Omega}\left(\mathbf{q}_{h}-\boldsymbol{\pi} \mathbf{q}\right) \cdot K\left(\mathbf{q}_{h}-\mathbf{q}\right) d \mathbf{x}$

$=\int_{\Omega}\left(\mathbf{q}_{h}-\boldsymbol{\pi} \mathbf{q}\right) \cdot K\left(\mathbf{q}_{h}-\boldsymbol{\pi} \mathbf{q}\right) d \mathbf{x}+\int_{\Omega}\left(\mathbf{q}_{h}-\boldsymbol{\pi} \mathbf{q}\right) \cdot K(\boldsymbol{\pi} \mathbf{q}-\mathbf{q}) d \mathbf{x}$,

$i v) \sum_{\mathcal{K} \in \mathcal{T}_{h}} \int_{\mathcal{K}} \nabla \theta_{u} \cdot e_{K \mathbf{q}} d \mathbf{x}-\sum_{F \in \mathcal{F}_{\mathrm{I}}} \int_{F} \llbracket \theta_{u} \rrbracket \cdot\left\{\left[e_{K \mathbf{q}}\right]\right\} d \sigma-\sum_{F \in \mathcal{F}_{\mathrm{D}}} \int_{F} \theta_{u} e_{K \mathbf{q}} \cdot \boldsymbol{\nu} d \sigma$

$$
=-\sum_{\mathcal{K} \in \mathcal{T}_{h}} \underbrace{\int_{\mathcal{K}} \theta_{u} \nabla \cdot e_{K \mathbf{q}} d \mathbf{x}}_{=0}+\sum_{F \in \mathcal{F}_{\mathrm{I}}} \int_{F}\left\{\left[\theta_{u} \rrbracket \rrbracket \llbracket e_{K \mathbf{q}} \rrbracket d \sigma+\sum_{F \in \mathcal{F}_{\mathrm{N}}} \int_{F} \theta_{u} e_{K \mathbf{q}} \cdot \boldsymbol{\nu} d \sigma\right.\right.
$$

and collecting terms we arrive at the error equation used for our estimate

$$
\begin{aligned}
& \frac{1}{2} \partial_{t} \int_{\Omega}\left(e_{u}\right)^{2} d \mathbf{x}+\int_{\Omega} e_{\mathbf{q}} \cdot K e_{\mathbf{q}} d \mathbf{x}+\eta \sum_{F \in \mathcal{F}_{\mathrm{I}}} h_{F} \int_{F}\left\|\llbracket e_{u} \rrbracket\right\|_{L^{2}(F)^{d}}^{2} d \sigma \\
& \quad+\eta \sum_{F \in \mathcal{F}_{\mathrm{D}}} h_{F}\left\|e_{u}\right\|_{L^{2}(F)}^{2}+\mu \sum_{F \in \mathcal{F}_{\mathrm{I}}}\left\|\llbracket e_{K \mathbf{q}}\right\|_{L^{2}(F)}^{2}+\mu \sum_{F \in \mathcal{F}_{\mathrm{N}}}\left\|e_{K \mathbf{q}}\right\|_{L^{2}(F)^{d}}^{2}
\end{aligned}
$$




$$
\begin{aligned}
= & -\underbrace{\int_{\Omega} e_{\mathbf{q}} \cdot K \theta_{\mathbf{q}} d \mathbf{x}}_{=: \Xi_{1}}-\underbrace{\eta \sum_{F \in \mathcal{F}_{I}} h_{F} \int_{F} \llbracket \theta_{u} \rrbracket \cdot \llbracket e_{u} \rrbracket d \sigma}_{=: \Xi_{2}}-\underbrace{\eta \sum_{F \in \mathcal{F}_{\mathrm{D}}} h_{F} \int_{F} \theta_{u} e_{u} d \sigma}_{=: \Xi_{3}}(6) \\
& -\underbrace{\sum_{F \in \mathcal{F}_{\mathrm{I}}} \int_{F}\left\{\left[\theta_{u}\right\rceil\right\} \llbracket e_{K \mathbf{q}} \rrbracket d \sigma}_{=: \Xi_{4}}-\underbrace{\sum_{F \in \mathcal{F}_{\mathrm{N}}} \int_{F} \theta_{u} e_{K \mathbf{q}} \cdot \boldsymbol{\nu} d \sigma}_{=: \Xi_{5}}-\underbrace{\sum_{F \in \mathcal{F}_{\mathrm{I}}} \int_{F}\left\{\left[\theta_{K \mathbf{q}} \rrbracket\right\} \llbracket \llbracket e_{u} \rrbracket d \sigma\right.}_{=: \Xi_{6}} \\
& -\underbrace{\sum_{F \in \mathcal{F}_{\mathrm{D}}} \int_{F} \theta_{K \mathbf{q}} \cdot \boldsymbol{\nu} e_{u} d \sigma}_{=: \Xi_{7}}-\mu \underbrace{\mu \sum_{F \in \mathcal{F}_{\mathrm{I}}} \int_{F} \llbracket \theta_{K \mathbf{q}} \rrbracket \llbracket e_{K \mathbf{q}} \rrbracket d \sigma}_{=: \Xi_{8}}-\underbrace{\mu \sum_{F \in \mathcal{F}_{\mathrm{N}}} \int_{F} \theta_{K \mathbf{q}} \cdot \boldsymbol{\nu} e_{K \mathbf{q}} \cdot \boldsymbol{\nu} d \sigma}_{=: \Xi_{9}} .
\end{aligned}
$$

Estimating $\Xi_{1}, \ldots, \Xi_{9}$ gives us

$$
\begin{aligned}
& \left|\Xi_{1}\right| \leq \frac{1}{2}\left\|\sqrt{K} e_{\mathbf{q}}\right\|_{L^{2}(\Omega)^{d}}^{2}+\underbrace{\frac{1}{2}\left\|\sqrt{K} \theta_{\mathbf{q}}\right\|_{L^{2}(\Omega)^{d}}^{2}}, \\
& =\mathcal{O}\left(h^{2 k+2}\right)\left[\text { Rem. 1, } L^{2} \text {-projection error }\right] \\
& \left|\Xi_{2}\right| \leq \frac{\eta}{4} \sum_{F \in \mathcal{F}_{\mathrm{I}}} h_{F}\left\|\llbracket e_{u} \rrbracket\right\|_{L^{2}(F)^{d}}^{2}+\underbrace{\eta \sum_{F \in \mathcal{F}_{\mathrm{I}}} h_{F}\left\|\llbracket \theta_{u} \rrbracket\right\|_{L^{2}(F)^{d}}}_{=\mathcal{O}\left(h^{2 k+2}\right)[\underline{\mathbf{P T I}}]} \\
& \left|\Xi_{3}\right| \leq \frac{\eta}{4} \sum_{F \in \mathcal{F}_{\mathrm{D}}} h_{F}\left\|\llbracket e_{u} \rrbracket\right\|_{L^{2}(F)^{d}}^{2}+\mathcal{O}\left(h^{2 k+2}\right),\left[\text { same as } \Xi_{2}\right] \\
& \left|\Xi_{4}\right| \leq \frac{\mu}{4} \sum_{F \in \mathcal{F}_{\mathrm{I}}}\left\|\llbracket e_{K \mathbf{q}} \rrbracket\right\|_{L^{2}(F)}^{2}+\underbrace{\frac{1}{\mu} \sum_{F \in \mathcal{F}_{\mathrm{I}}}\left\|\left\{\left[\theta_{u}\right]\right\}\right\|_{L^{2}(F)}^{2}}_{=\mathcal{O}\left(h^{2 k+1}\right)[\underline{\mathbf{P T I}}]} \\
& \left.\left|\Xi_{5}\right| \leq \frac{\mu}{4} \sum_{F \in \mathcal{F}_{\mathrm{N}}}\left\|e_{K \mathbf{q}}\right\|_{L^{2}(F)^{d}}^{2}+\mathcal{O}\left(h^{2 k+1}\right), \text { [same as } \Xi_{4}\right] \\
& \left|\Xi_{6}\right| \leq \frac{\eta}{4} \sum_{F \in \mathcal{F}_{\mathrm{I}}} h_{F}\left\|\llbracket e_{u} \rrbracket\right\|_{L^{2}(F)^{d}}^{2}+\underbrace{\frac{1}{\eta} \sum_{F \in \mathcal{F}_{\mathrm{I}}} \frac{1}{h_{F}}\left\|\left\{\left[\theta_{K \mathbf{q}}\right]\right\} \cdot \boldsymbol{\nu}\right\|_{L^{2}(F)}^{2},}_{=\mathcal{O}\left(h^{2 k}\right)\left[K \in W^{k+1, \infty}(\Omega)^{d, d}, \underline{\mathbf{P T I}}\right]} \\
& \left.\left|\Xi_{7}\right| \leq \frac{\eta}{4} \sum_{F \in \mathcal{F}_{\mathrm{D}}} h_{F}\left\|e_{u}\right\|_{L^{2}(F)}^{2}+\mathcal{O}\left(h^{2 k}\right), \text { [same as } \Xi_{6}\right] \\
& \left|\Xi_{8}\right| \leq \frac{\mu}{4} \sum_{F \in \mathcal{F}_{\mathrm{I}}}\left\|\llbracket e_{K \mathbf{q}} \rrbracket\right\|_{L^{2}(F)}^{2}+\underbrace{\mu \sum_{F \in \mathcal{F}_{\mathrm{I}}}\left\|\llbracket \theta_{K \mathbf{q}} \rrbracket\right\|_{L^{2}(F)}^{2}}_{=\mathcal{O}\left(h^{2 k+1}\right)[\underline{\mathbf{P T I}}]}, \\
& \left|\Xi_{9}\right| \leq \frac{\mu}{4} \sum_{F \in \mathcal{F}_{\mathrm{N}}}\left\|e_{K \mathbf{q}}\right\|_{L^{2}(F)^{d}}^{2}+\mathcal{O}\left(h^{2 k+1}\right) \cdot\left[\text { same as } \Xi_{8}\right]
\end{aligned}
$$

Substituting $\Xi_{1}, \ldots, \Xi_{9}$ into (6), integrating over time, and using the triangle inequality produces the error estimate. 


\section{Numerical results}

In this section, numerical results illustrating the method's performance are presented. To better demonstrate the potential of our scheme, we use in Section $4.1 \mathrm{~K}$ that is not symmetric; in Section 4.2 , a discontinuous $K$ is tested. These two cases treat a stationary equivalent of (1), i.e., the system

$$
\left\{\begin{aligned}
\nabla \cdot(K \mathbf{q}) & =f & & \text { in } \Omega, \\
\mathbf{q}+\nabla u & =0 & & \text { in } \Omega, \\
u & =u_{D} & & \text { on } \Gamma_{\mathrm{D}} .
\end{aligned}\right.
$$

The extension of the stationary case to the unsteady case can be easily performed by using, e.g., diagonally implicit Runge-Kutta (DIRK) formula. In this work, we choose trapezoidal rule for this purpose. Numerical results are shown in Section 4.3 for a time-dependent diffusion problem with a symmetric $K$ and smooth solution; in Section 4.4, we attempt to quantify the effect of penalties on the numerical diffusion by comparing results of our scheme and a 'standard' LDG formulation -- assumed there to have both penalty coefficients independent of the mesh size $h$. Finally, Section 4.5 is dedicated to the verification of convergence results using a more complicated domain and an unstructured mesh - once again, the results are compared to the 'standard' LDG.

\subsection{Smooth permeability coefficient}

The permeability coefficient

$$
K(x, y)=\left(\begin{array}{cc}
e^{y / 5} & 1 / 2 \\
1 / 3 & e^{x / 5}
\end{array}\right)
$$

is not symmetric but positive definite in the ranges considered here. The domain is set to $\Omega:=[0,1]^{2}$, and $f$ is chosen in such a way that the function

$$
u(x, y)=\cos (3 x) \cos (3 y)
$$

is indeed a solution. In Table 1 and Figure 1, we plot and show, respectively, the convergence results for $u$ (left) and $\mathbf{q}$ (right) for different polynomial orders $k$. The errors are measured in the $L^{2}$-norm on domain $\Omega$. In all cases, the convergence orders of the primary unknown $u$ and the flux unknown $\mathbf{q}$ are optimal and thus exceed the expectations based on the results of our a priori analysis. In some sense, this is in good agreement with the results in [8]; however, those do not cover our choice of stabilization parameters.

The presented method and its analysis make extensive use of the $L^{2}$-projection for the flux unknown, e.g., $\boldsymbol{\pi}\left(K \mathbf{q}_{h}\right)$. This projection needed to simplify analysis is, however, tedious to implement. Using our scheme without this projection can be seen as a non-perfect way of integrating the equations, i.e., there would be an additional truncation error for the computation of approximate solutions 


\begin{tabular}{|l|l|l|l|l|l|}
\hline Polynomial order & \# Elements & $\left\|u-u_{h}\right\|_{L^{2}(\Omega)}$ & RoC & $\left\|\nabla u+\mathbf{q}_{h}\right\|_{L^{2}(\Omega)}$ & RoC \\
\hline & 8 & $1.12 \mathrm{E}+00$ & & $1.05 \mathrm{E}+00$ & \\
0 & 32 & $8.27 \mathrm{E}-01$ & 0.44 & $4.49 \mathrm{E}-01$ & 1.23 \\
& 128 & $4.29 \mathrm{E}-01$ & 0.95 & $2.29 \mathrm{E}-01$ & 0.97 \\
& 512 & $2.08 \mathrm{E}-01$ & 1.05 & $1.12 \mathrm{E}-01$ & 1.03 \\
& 2048 & $1.00 \mathrm{E}-01$ & 1.05 & $5.42 \mathrm{E}-02$ & 1.05 \\
\hline \multirow{5}{*}{1} & 8 & $3.61 \mathrm{E}-01$ & & $3.86 \mathrm{E}-01$ & \\
& 32 & $1.02 \mathrm{E}-01$ & 1.83 & $9.76 \mathrm{E}-02$ & 1.99 \\
& 128 & $3.29 \mathrm{E}-02$ & 1.63 & $2.66 \mathrm{E}-02$ & 1.88 \\
& 512 & $1.00 \mathrm{E}-02$ & 1.72 & $7.13 \mathrm{E}-03$ & 1.90 \\
& 2048 & $2.90 \mathrm{E}-03$ & 1.79 & $1.88 \mathrm{E}-03$ & 1.92 \\
\hline \multirow{5}{*}{3} & 8 & $7.22 \mathrm{E}-02$ & & $8.24 \mathrm{E}-02$ & \\
& 32 & $1.01 \mathrm{E}-02$ & 2.84 & $1.04 \mathrm{E}-02$ & 2.99 \\
& 128 & $1.53 \mathrm{E}-03$ & 2.72 & $1.41 \mathrm{E}-03$ & 2.88 \\
& 512 & $2.23 \mathrm{E}-04$ & 2.78 & $1.88 \mathrm{E}-04$ & 2.90 \\
& 2048 & $3.08 \mathrm{E}-05$ & 2.86 & $2.48 \mathrm{E}-05$ & 2.93 \\
\hline \multirow{5}{*}{4} & 8 & $1.26 \mathrm{E}-02$ & & $1.38 \mathrm{E}-02$ & \\
& 32 & $8.11 \mathrm{E}-04$ & 3.96 & $8.64 \mathrm{E}-04$ & 4.00 \\
& 128 & $6.10 \mathrm{E}-05$ & 3.73 & $5.83 \mathrm{E}-05$ & 3.89 \\
& 512 & $4.24 \mathrm{E}-06$ & 3.85 & $3.84 \mathrm{E}-06$ & 3.92 \\
& 2048 & $2.83 \mathrm{E}-07$ & 3.90 & $2.48 \mathrm{E}-07$ & 3.96 \\
\hline \multirow{2}{*}{} & 8 & $1.70 \mathrm{E}-03$ & & $1.89 \mathrm{E}-03$ & \\
& 32 & $5.38 \mathrm{E}-05$ & 4.98 & $5.88 \mathrm{E}-05$ & 5.00 \\
& 128 & $2.05 \mathrm{E}-06$ & 4.71 & $1.99 \mathrm{E}-06$ & 4.88 \\
& 512 & $7.50 \mathrm{E}-08$ & 4.77 & $6.65 \mathrm{E}-08$ & 4.91 \\
& 2048 & $2.60 \mathrm{E}-09$ & 4.85 & $2.18 \mathrm{E}-09$ & 4.93 \\
\hline
\end{tabular}

Table 1 Smooth permeability coefficient: Errors for primary $\left\|u-u_{h}\right\|_{L^{2}(\Omega)}$ and flux $\| \nabla u+$ $\mathbf{q}_{h} \|_{L^{2}(\Omega)}$ variables and rates of convergence (RoC). Optimal orders can be observed for both unknowns.
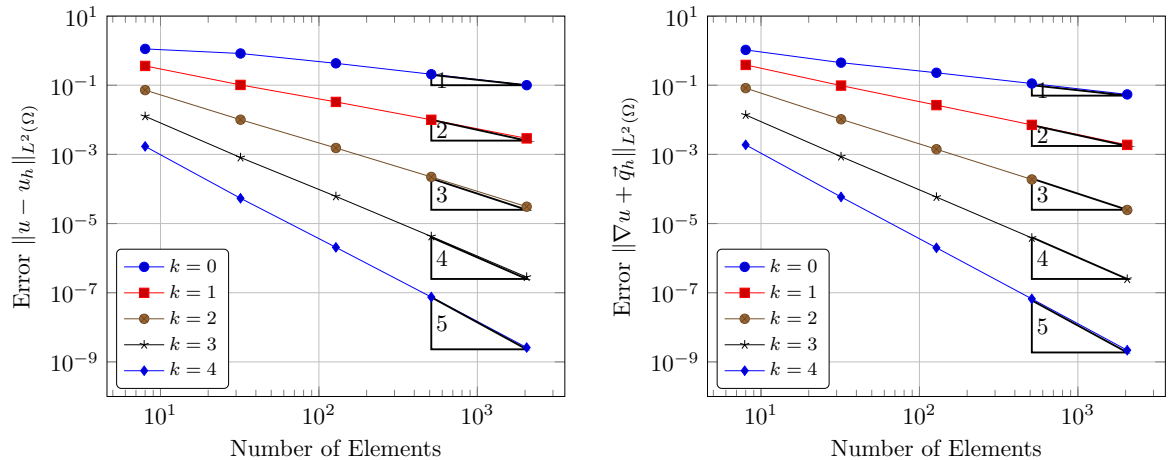

Fig. 1 Smooth permeability coefficient: Convergence plots for primary $\left\|u-u_{h}\right\|_{L^{2}(\Omega)}$ and flux $\left\|\nabla u+\mathbf{q}_{h}\right\|_{L^{2}(\Omega)}$ variables. Note the optimal convergence orders for both unknowns.

$u_{h}, \mathbf{q}_{h}$. In order to gauge the effect of projection in practical computations, we compared results with and without the projection. In Table 2, errors for $k=3$ for both cases are documented exemplarily. The results clearly indicate that it does not matter whether to use the projection or not. Similar results were obtained for all other polynomial orders. 


\begin{tabular}{|l|l|l|}
\hline Number of elements & $\left\|u-u_{h}\right\|_{L^{2}(\Omega)}$, not using $\boldsymbol{\pi}$ & $\left\|u-u_{h}\right\|_{L^{2}(\Omega)}$, using $\boldsymbol{\pi}$ \\
\hline 8 & $\underline{0.0125} 901$ & $\underline{0.0126056}$ \\
32 & $\underline{0.000811} 133$ & $\underline{0.000811091}$ \\
128 & $\underline{6.10577 \mathrm{e}-05}$ & $\underline{6.10205 \mathrm{e}-05}$ \\
512 & $\underline{4.24} 131 \mathrm{e}-06$ & $\underline{4.23} 792 \mathrm{e}-06$ \\
\hline
\end{tabular}

Table 2 Smooth permeability coefficient: Is it necessary to use the $L^{2}$-projection operator for boundary fluxes? Errors for $k=3$ shown above indicate that it is not needed - similarly to all other results of our numerical studies. Underlined digits are equal up to rounding.

\subsection{Discontinuous permeability coefficient}

In this section, we modify the above test to include a discontinuous permeability. Coefficient $K-$ similarly to Section $4.1-$ is defined as

$$
K(x, y)=h(x)\left(\begin{array}{cc}
e^{y / 5} & \frac{1}{2} \\
\frac{1}{3} & e^{x / 5}
\end{array}\right), \quad \text { with } h(x):= \begin{cases}\frac{1}{1000}, & x<0 \\
1, & x \geq 0\end{cases}
$$

chosen to produce a discontinuity (jump of three orders of magnitude) in the coefficient. This time, we consider the domain $\Omega:=[-1,1] \times[0,1]$. The exact solution to the PDE with $f$ chosen accordingly is given by

$$
u(x, y)=e^{-x^{2} y} .
$$

Note that by construction, this solution fulfills $\llbracket K \mathbf{q} \rrbracket=0$. Numerical results are shown in Table 3 and Figure 2. Due to the low regularity of the problem, one cannot expect optimal orders of convergence, but it is still clearly visible that a higher polynomial degree $k$ indeed yields an improvement over a lower one - of course, at additional computational cost.
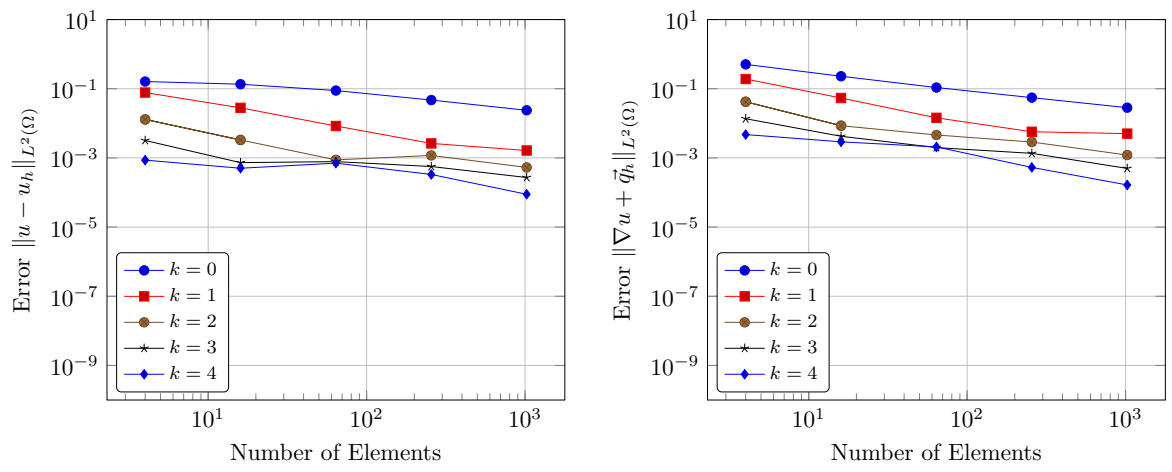

Fig. 2 Discontinuous permeability coefficient: Convergence plots for primary $\left\|u-u_{h}\right\|_{L^{2}(\Omega)}$ and flux $\left\|\nabla u+\mathbf{q}_{h}\right\|_{L^{2}(\Omega)}$ variables. 


\begin{tabular}{|l|l|l|l|l|l|}
\hline Polynomial order & \# Elements & $\left\|u-u_{h}\right\|_{L^{2}(\Omega)}$ & RoC & $\left\|\nabla u+\mathbf{q}_{h}\right\|_{L^{2}(\Omega)}$ & RoC \\
\hline & 4 & $1.62 \mathrm{E}-01$ & & $5.06 \mathrm{E}-01$ & \\
0 & 16 & $1.35 \mathrm{E}-01$ & 0.26 & $2.30 \mathrm{E}-01$ & 1.14 \\
& 64 & $8.86 \mathrm{E}-02$ & 0.61 & $1.08 \mathrm{E}-01$ & 1.09 \\
& 256 & $4.71 \mathrm{E}-02$ & 0.91 & $5.54 \mathrm{E}-02$ & 0.97 \\
& 1024 & $2.38 \mathrm{E}-02$ & 0.98 & $2.84 \mathrm{E}-02$ & 0.97 \\
\hline \multirow{5}{*}{1} & 4 & $7.76 \mathrm{E}-02$ & & $1.91 \mathrm{E}-01$ & \\
& 16 & $2.80 \mathrm{E}-02$ & 1.47 & $5.40 \mathrm{E}-02$ & 1.82 \\
& 64 & $8.38 \mathrm{E}-03$ & 1.74 & $1.44 \mathrm{E}-02$ & 1.90 \\
& 256 & $2.63 \mathrm{E}-03$ & 1.67 & $5.71 \mathrm{E}-03$ & 1.34 \\
& 1024 & $1.64 \mathrm{E}-03$ & 0.68 & $5.04 \mathrm{E}-03$ & 0.18 \\
\hline \multirow{5}{*}{3} & 16 & $1.30 \mathrm{E}-02$ & & $4.20 \mathrm{E}-02$ & \\
& 64 & $3.30 \mathrm{E}-03$ & 1.97 & $8.54 \mathrm{E}-03$ & 2.30 \\
& 256 & $8.79 \mathrm{E}-04$ & 1.91 & $4.61 \mathrm{E}-03$ & 0.89 \\
& 1024 & $1.18 \mathrm{E}-03$ & -0.42 & $2.88 \mathrm{E}-03$ & 0.68 \\
& 4 & $5.31 \mathrm{E}-04$ & 1.15 & $1.21 \mathrm{E}-03$ & 1.25 \\
\hline \multirow{5}{*}{4} & 16 & $3.19 \mathrm{E}-03$ & & $1.36 \mathrm{E}-02$ & \\
& 64 & $7.30 \mathrm{E}-04$ & 2.13 & $4.22 \mathrm{E}-03$ & 1.69 \\
& 256 & $7.81 \mathrm{E}-04$ & -0.10 & $1.99 \mathrm{E}-03$ & 1.09 \\
& 1024 & $5.64 \mathrm{E}-04$ & 0.47 & $1.35 \mathrm{E}-03$ & 0.55 \\
& 4 & $2.71 \mathrm{E}-04$ & 1.06 & $4.97 \mathrm{E}-04$ & 1.45 \\
\hline
\end{tabular}

Table 3 Discontinuous permeability coefficient: Errors for primary $\left\|u-u_{h}\right\|_{L^{2}(\Omega)}$ and flux $\left\|\nabla u+\mathbf{q}_{h}\right\|_{L^{2}(\Omega)}$ variables and rates of convergence (RoC). Although the problem's regularity is too low for optimal convergence, lower errors are observed for higher order polynomials.

\subsection{Time-dependent test}

In this section, we discuss the time-dependent problem on domain $\Omega=[0,1]^{2}$,

$$
\left\{\begin{aligned}
u_{t}+\nabla \cdot(K \mathbf{q}) & =f & & \text { in } \Omega, \\
\mathbf{q}+\nabla u & =0 & & \text { in } \Omega, \\
u & =u_{D} & & \text { on } \Gamma_{\mathrm{D}} .
\end{aligned}\right.
$$

for the symmetric positive definite matrix $K$ given by

$$
K(x, y)=\left(\begin{array}{cc}
e^{y / 5} & 1 / 2 \\
1 / 2 & e^{x / 5}
\end{array}\right)
$$

Source term $f$ and boundary conditions $u_{D}$ are set in such a way that the exact solution is given by

$$
u(x, y, t)=\cos (x+t) \cos (y+t)
$$

final time $T_{\text {end }}$ is defined to be 0.5 . For each refinement level, we choose $\Delta t$ small enough such that the error in each step is independent of time. For the above test problem, this time step turned out to be equal to 0.01 for the coarsest mesh (8 Elements), this time step was then divided by four for 
each successive refinement level. Convergence results are listed in Table 4 and plotted in Figure 3. Again, the algorithm converges with the optimal orders in both $u$ and $q$, which means that the orders exceed the theoretical predictions. To illustrate the behavior of our scheme with regard to the time discretization, we consider the same error norms at $T_{\text {end }}=0.5$ on the grid with 128 elements. To make this task more challenging approximation orders $p=3$ and $p=4$ are used; errors and convergence orders for different time step sizes are given in Table 5 .

\begin{tabular}{|l|l|l|l|l|l|}
\hline Polynomial order & \# Elements & $\left\|u-u_{h}\right\|_{L^{2}(\Omega)}$ & $\mathrm{RoC}$ & $\left\|\nabla u+\mathbf{q}_{h}\right\|_{L^{2}(\Omega)}$ & RoC \\
\hline \multirow{3}{*}{0} & 8 & $1.22 \mathrm{E}-01$ & & $1.43 \mathrm{E}-01$ & \\
& 32 & $4.55 \mathrm{E}-02$ & 1.42 & $1.98 \mathrm{E}-01$ & -0.48 \\
& 128 & $2.34 \mathrm{E}-02$ & 0.96 & $1.37 \mathrm{E}-01$ & 0.53 \\
1 & 512 & $1.16 \mathrm{E}-02$ & 1.01 & $8.90 \mathrm{E}-02$ & 0.62 \\
\hline \multirow{3}{*}{2} & 8 & $2.76 \mathrm{E}-02$ & & $3.40 \mathrm{E}-02$ & \\
& 32 & $4.22 \mathrm{E}-03$ & 2.71 & $5.66 \mathrm{E}-03$ & 2.59 \\
& 128 & $1.47 \mathrm{E}-03$ & 1.52 & $1.76 \mathrm{E}-03$ & 1.69 \\
& 512 & $4.41 \mathrm{E}-04$ & 1.73 & $4.90 \mathrm{E}-04$ & 1.84 \\
\hline & 8 & $9.19 \mathrm{E}-04$ & & $1.19 \mathrm{E}-03$ & \\
& 32 & $1.42 \mathrm{E}-04$ & 2.70 & $1.75 \mathrm{E}-04$ & 2.77 \\
& 128 & $2.04 \mathrm{E}-05$ & 2.80 & $2.46 \mathrm{E}-05$ & 2.83 \\
& 512 & $2.79 \mathrm{E}-06$ & 2.87 & $3.27 \mathrm{E}-06$ & 2.91 \\
\hline
\end{tabular}

Table 4 Time-dependent test case: Errors for primary $\left\|u-u_{h}\right\|_{L^{2}(\Omega)}$ and flux $\| \nabla u+$ $\mathbf{q}_{h} \|_{L^{2}(\Omega)}$ variables and rates of convergence (RoC).
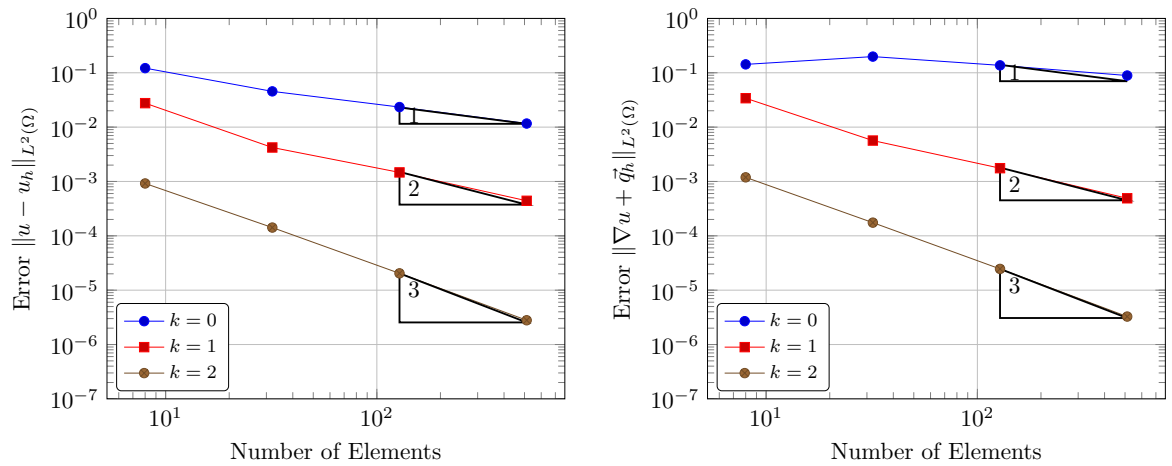

Fig. 3 Time-dependent test case: Convergence plots for primary $\left\|u-u_{h}\right\|_{L^{2}(\Omega)}$ and flux $\left\|\nabla u+\mathbf{q}_{h}\right\|_{L^{2}(\Omega)}$ variable. Error is measured at time $T_{\text {end }}=0.5$.

4.4 Quantifying the numerical diffusion

Since one of the main ideas behind our DG formulation is to reduce the amount of numerical diffusion introduced by the penalty terms, we attempt to quantify 


\begin{tabular}{|l|l|l|l|l|l|}
\hline Polynomial order & $\Delta t$ & $\left\|u-u_{h}\right\|_{L^{2}(\Omega)}$ & RoC & $\left\|\nabla u+\mathrm{q}_{h}\right\|_{L^{2}(\Omega)}$ & RoC \\
\hline \multirow{5}{*}{3} & 0.1 & $1.15 \mathrm{E}-04$ & & $5.28 \mathrm{E}-04$ & \\
& 0.05 & $2.86 \mathrm{E}-05$ & 2.01 & $1.30 \mathrm{E}-04$ & 2.02 \\
& 0.025 & $7.17 \mathrm{E}-06$ & 2.00 & $3.26 \mathrm{E}-05$ & 2.00 \\
& 0.0125 & $1.84 \mathrm{E}-06$ & 1.96 & $8.16 \mathrm{E}-06$ & 2.00 \\
& 0.00625 & $6.11 \mathrm{E}-07$ & 1.59 & $2.08 \mathrm{E}-06$ & 1.97 \\
& $3.13 \mathrm{E}-03$ & $4.31 \mathrm{E}-07$ & 0.50 & $6.65 \mathrm{E}-07$ & 1.65 \\
& $1.56 \mathrm{E}-03$ & $4.17 \mathrm{E}-07$ & 0.05 & $4.45 \mathrm{E}-07$ & 0.58 \\
& $7.81 \mathrm{E}-04$ & $4.16 \mathrm{E}-07$ & 0.00 & $4.28 \mathrm{E}-07$ & 0.06 \\
& $3.91 \mathrm{E}-04$ & $4.16 \mathrm{E}-07$ & 0.00 & $4.27 \mathrm{E}-07$ & 0.00 \\
& $1.95 \mathrm{E}-04$ & $4.16 \mathrm{E}-07$ & 0.00 & $4.27 \mathrm{E}-07$ & 0.00 \\
& $9.77 \mathrm{E}-05$ & $4.16 \mathrm{E}-07$ & 0.00 & $4.27 \mathrm{E}-07$ & 0.00 \\
& $4.88 \mathrm{E}-05$ & $4.16 \mathrm{E}-07$ & 0.00 & $4.27 \mathrm{E}-07$ & 0.00 \\
\hline \multirow{5}{*}{4} & 0.1 & $1.15 \mathrm{E}-04$ & & $5.28 \mathrm{E}-04$ & \\
& 0.05 & $2.86 \mathrm{E}-05$ & 2.01 & $1.30 \mathrm{E}-04$ & 2.02 \\
& 0.025 & $7.16 \mathrm{E}-06$ & 2.00 & $3.26 \mathrm{E}-05$ & 2.00 \\
& 0.0125 & $1.79 \mathrm{E}-06$ & 2.00 & $8.15 \mathrm{E}-06$ & 2.00 \\
& 0.00625 & $4.47 \mathrm{E}-07$ & 2.00 & $2.04 \mathrm{E}-06$ & 2.00 \\
& $3.13 \mathrm{E}-03$ & $1.12 \mathrm{E}-07$ & 2.00 & $5.10 \mathrm{E}-07$ & 2.00 \\
& $1.56 \mathrm{E}-03$ & $2.81 \mathrm{E}-08$ & 1.99 & $1.27 \mathrm{E}-07$ & 2.00 \\
& $7.81 \mathrm{E}-04$ & $7.68 \mathrm{E}-09$ & 1.87 & $3.20 \mathrm{E}-08$ & 1.99 \\
& $3.91 \mathrm{E}-04$ & $3.64 \mathrm{E}-09$ & 1.08 & $8.52 \mathrm{E}-09$ & 1.91 \\
& $1.95 \mathrm{E}-04$ & $3.22 \mathrm{E}-09$ & 0.18 & $3.63 \mathrm{E}-09$ & 1.23 \\
& $9.77 \mathrm{E}-05$ & $3.19 \mathrm{E}-09$ & 0.01 & $3.08 \mathrm{E}-09$ & 0.24 \\
& $4.88 \mathrm{E}-05$ & $3.19 \mathrm{E}-09$ & 0.00 & $3.04 \mathrm{E}-09$ & 0.02 \\
\hline
\end{tabular}

Table 5 Time-dependent test case: Errors for primary $\left\|u-u_{h}\right\|_{L^{2}(\Omega)}$ and flux $\| \nabla u+$ $\mathbf{q}_{h} \|_{L^{2}(\Omega)}$ variables on the mesh with 128 elements and rates of convergence (RoC) in time.

the effect of penalties by selecting an appropriate problem and comparing the results to a 'standard' LDG scheme.

As a test problem we choose an inverted paraboloid of height one that is slowly diffused with the time. As a measure of numerical diffusion we focus on the maximum (corresponding to the error at the apex) and the minimum (indicating the amount of 'leakage' at the base) values of the discrete solution. The initial state on domain $\Omega=[0,1]^{2}$ shown in Figure 4 (left) is specified by

$$
u_{0}= \begin{cases}1-9\left(\left(x-\frac{1}{2}\right)^{2}+\left(y-\frac{1}{2}\right)^{2}\right), & \left(x-\frac{1}{2}\right)^{2}+\left(y-\frac{1}{2}\right)^{2} \leq \frac{1}{9} \\ 0, & \text { otherwise }\end{cases}
$$

and the permeability coefficient is set to

$$
K(x, y)=\left(\begin{array}{cc}
0.01 & 0 \\
0 & 0.01
\end{array}\right)
$$

The final state as given by the piecewise quadratic LDG solution on the mesh with 512 elements is plotted in Figure 4 (right). The latter solution is considered as fully converged and serves as the reference.

For comparison purposes, the penalty coefficients in the Finite Element Problem are set to $\eta=\mu=1$. The LDG scheme chosen as a reference uses the same values of $\eta$ and $\mu$ but without any dependency on $h$. This implies a comparison 

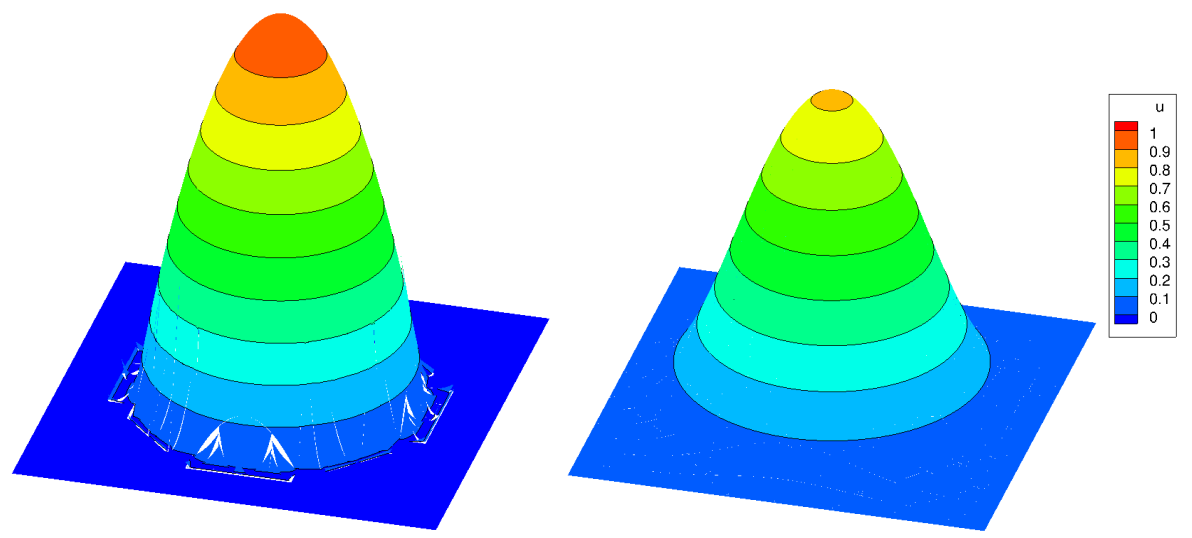

Fig. 4 Paraboloid diffusion problem: Initial condition (left), final state (right).

between $\llbracket u_{h} \rrbracket$ penalty coefficients of $h$ (our scheme) and 1 (LDG), whereas the second (flux) penalty is exactly the same in both schemes.

The solution extrema at the final time for all computed cases are listed in Table 6. For piecewise linear and quadratic approximations, very little difference between extremal values for both formulations can be detected (the same also holds for the solutions themselves). However, the piecewise constant approximations - well known to be much more dependent on a specific flux approximation - clearly demonstrate the advantages of using smaller penalties. This benefit does not only apply to the extrema of the scalar field, but can also be detected in the general solution behavior as shown exemplarily in Figures 5, 6. Such behavior suggests that the specific choice of penalties in our scheme could prove particularly advantageous for advection dominated advection-diffusion problems.
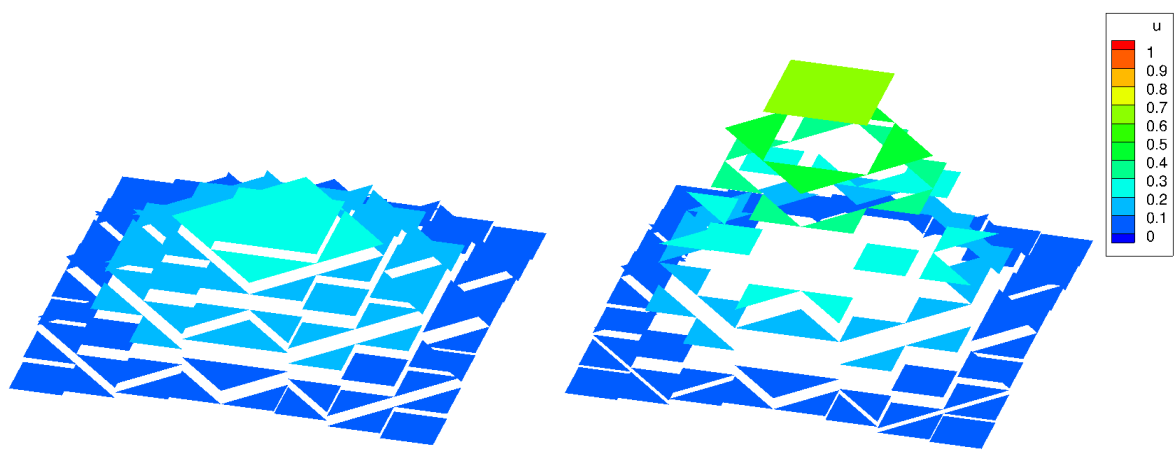

Fig. 5 Paraboloid diffusion problem: Final state for piecewise constant discretization on the mesh with 128 elements. LDG (left), our scheme (right). 

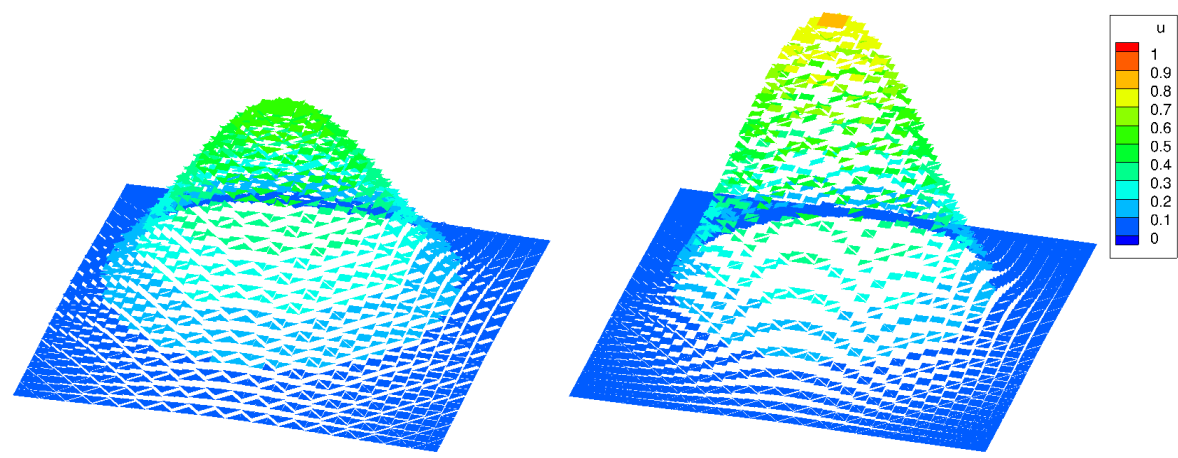

Fig. 6 Paraboloid diffusion problem: Final state for piecewise constant discretization on the mesh with 2048 elements. LDG (left), our scheme (right).

\begin{tabular}{|l|l||l|l||l|l|}
\hline \multirow{2}{*}{$\begin{array}{l}\text { Polynomial } \\
\text { order }\end{array}$} & \multicolumn{2}{|c||}{ \# Elements } & $u_{\text {min }}$ & $u_{\text {max }}$ & \multicolumn{2}{c|}{ Our scheme } \\
\hline \multirow{5}{*}{0} & 8 & $1.63 \mathrm{E}-02$ & $2.89 \mathrm{E}-02$ & $4.59 \mathrm{E}-02$ & $6.60 \mathrm{E}-02$ \\
& 32 & $2.75 \mathrm{E}-02$ & $1.23 \mathrm{E}-01$ & $3.16 \mathrm{E}-02$ & $3.10 \mathrm{E}-01$ \\
& 128 & $1.30 \mathrm{E}-02$ & $2.50 \mathrm{E}-01$ & $3.27 \mathrm{E}-03$ & $6.04 \mathrm{E}-01$ \\
& 512 & $3.34 \mathrm{E}-03$ & $4.05 \mathrm{E}-01$ & $2.16 \mathrm{E}-04$ & $7.55 \mathrm{E}-01$ \\
& 2048 & $4.85 \mathrm{E}-04$ & $5.53 \mathrm{E}-01$ & $1.91 \mathrm{E}-05$ & $8.02 \mathrm{E}-01$ \\
& 8192 & $4.89 \mathrm{E}-05$ & $6.68 \mathrm{E}-01$ & $2.83 \mathrm{E}-06$ & $8.16 \mathrm{E}-01$ \\
& 32768 & $4.45 \mathrm{E}-06$ & $7.39 \mathrm{E}-01$ & $5.41 \mathrm{E}-07$ & $8.19 \mathrm{E}-01$ \\
& 131072 & $4.75 \mathrm{E}-07$ & $7.79 \mathrm{E}-01$ & $1.20 \mathrm{E}-07$ & $8.20 \mathrm{E}-01$ \\
\hline \multirow{3}{*}{2} & 8 & $-7.65 \mathrm{E}-03$ & $6.48 \mathrm{E}-01$ & $-1.18 \mathrm{E}-02$ & $6.55 \mathrm{E}-01$ \\
& 32 & $-2.33 \mathrm{E}-04$ & $8.78 \mathrm{E}-01$ & $-7.76 \mathrm{E}-03$ & $8.78 \mathrm{E}-01$ \\
& 128 & $-2.03 \mathrm{E}-03$ & $8.46 \mathrm{E}-01$ & $-3.66 \mathrm{E}-03$ & $8.47 \mathrm{E}-01$ \\
& 512 & $-3.35 \mathrm{E}-05$ & $8.27 \mathrm{E}-01$ & $-1.61 \mathrm{E}-04$ & $8.28 \mathrm{E}-01$ \\
\hline & 8 & $-2.09 \mathrm{E}-02$ & $9.40 \mathrm{E}-01$ & $-2.14 \mathrm{E}-02$ & $9.38 \mathrm{E}-01$ \\
& 32 & $-9.12 \mathrm{E}-03$ & $8.58 \mathrm{E}-01$ & $-7.84 \mathrm{E}-03$ & $8.53 \mathrm{E}-01$ \\
& 128 & $-1.56 \mathrm{E}-04$ & $8.23 \mathrm{E}-01$ & $-8.87 \mathrm{E}-05$ & $8.21 \mathrm{E}-01$ \\
& 512 & $-1.65 \mathrm{E}-06$ & $8.21 \mathrm{E}-01$ & $2.83 \mathrm{E}-07$ & $8.21 \mathrm{E}-01$ \\
\hline
\end{tabular}

Table 6 Paraboloid diffusion problem: Min/max values of the solution at the final time $T_{\text {end }}=0.5$. Polynomial orders $k=0$ (top), $k=1$ (middle), $k=2$ (bottom).

\subsection{Unstructured grid test}

The goal of the last test case is to verify the convergence results and method's performance using a more complicated domain and an unstructured mesh. Here we consider a problem whose analytical solution is given by

$$
u(x, t)=\exp (-t) \cos (x+y+t)
$$

with the diffusion tensor $K$ represented by a unit matrix. Figure 7 illustrates the domain geometry, the coarsest mesh consisting of 10 elements, and the initial condition. In Table 7, we list the convergence rates for our scheme and the LDG method with the penalty terms defined as in the previous section. Figure 8 illustrates the convergence rates for our method. We see that all 
convergence rates are similar to structured grid cases, also the accuracy of our scheme is very much comparable to that of the standard LDG method.

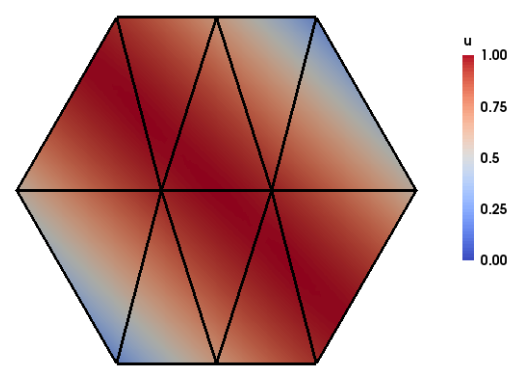

Fig. 7 Unstructured grid test: Initial condition and the coarsest mesh with 10 elements.
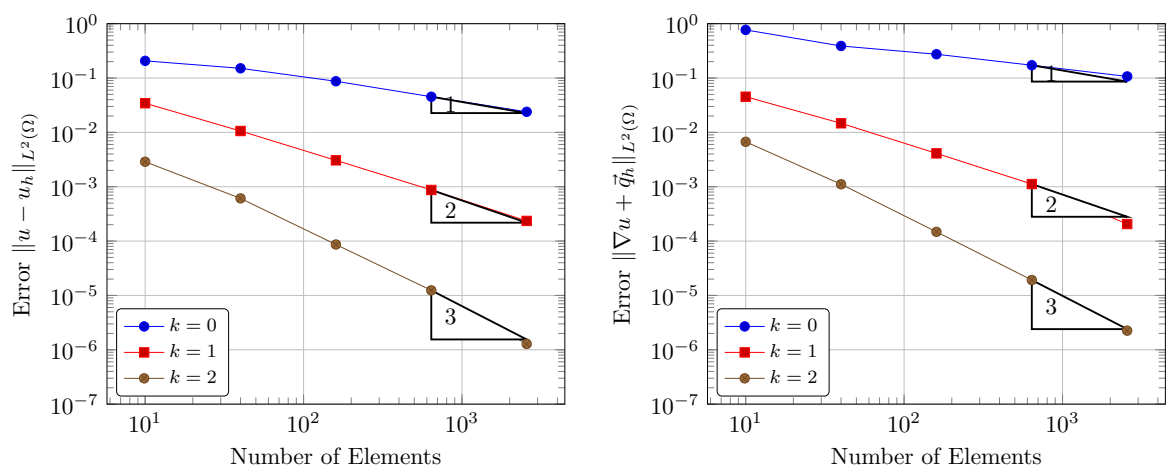

Fig. 8 Unstructured grid test: Convergence plots for primary $\left\|u-u_{h}\right\|_{L^{2}(\Omega)}$ and flux $\left\|\nabla u+\mathbf{q}_{h}\right\|_{L^{2}(\Omega)}$ variable. Error is measured at time $T_{\text {end }}=0.5$.

\section{Conclusions}

The results of our numerical studies suggest that the proposed scheme has the optimal convergence for both, the primary and the flux unknowns. This indicates some potential for improvement in our analysis. The reduced penalty coefficients (scaling as $O(h)$ ) appear to have no negative effects on stability, convergence, or accuracy of the DG discretization; however, significant improvements in the numerical diffusion properties of the method were detected in the lowest order case. This indicates a good potential of our formulation especially for advection dominated problems. 


\begin{tabular}{|c|c|c|c|c|c|}
\hline \multirow{2}{*}{$\begin{array}{l}\text { Polynomial } \\
\text { order }\end{array}$} & \multirow[b]{2}{*}{ \# Elements } & \multicolumn{2}{|l|}{ LDG } & \multicolumn{2}{|c|}{ Our scheme } \\
\hline & & $\left\|u-u_{h}\right\|_{L^{2}(\Omega)}$ & $\mathrm{RoC}$ & $\left\|u-u_{h}\right\|_{L^{2}(\Omega)}$ & RoC \\
\hline \multirow{5}{*}{0} & 10 & $1.72 \mathrm{E}-01$ & & $2.07 \mathrm{E}-01$ & \\
\hline & 40 & $9.65 \mathrm{E}-02$ & 0.83 & $1.51 \mathrm{E}-01$ & 0.45 \\
\hline & 160 & $5.44 \mathrm{E}-02$ & 0.83 & 8.72E-02 & 0.79 \\
\hline & 640 & 2.90E-02 & 0.90 & $4.53 \mathrm{E}-02$ & 0.95 \\
\hline & 2560 & $1.68 \mathrm{E}-02$ & 0.79 & 2.39E-02 & 0.92 \\
\hline \multirow{5}{*}{1} & 10 & $3.43 \mathrm{E}-02$ & & $3.44 \mathrm{E}-02$ & \\
\hline & 40 & 9.19E-03 & 1.90 & $1.06 \mathrm{E}-02$ & 1.69 \\
\hline & 160 & $2.27 \mathrm{E}-03$ & 2.02 & $3.06 \mathrm{E}-03$ & 1.80 \\
\hline & 640 & 5.61E-04 & 2.01 & 8.72E-04 & 1.81 \\
\hline & 2560 & $1.19 \mathrm{E}-04$ & 2.24 & $2.35 \mathrm{E}-04$ & 1.89 \\
\hline \multirow{5}{*}{2} & 10 & $2.65 \mathrm{E}-03$ & & $2.88 \mathrm{E}-03$ & \\
\hline & 40 & $5.25 \mathrm{E}-04$ & 2.34 & $6.10 \mathrm{E}-04$ & 2.24 \\
\hline & 160 & $6.83 \mathrm{E}-05$ & 2.94 & $8.65 \mathrm{E}-05$ & 2.82 \\
\hline & 640 & 8.72E-06 & 2.97 & $1.24 \mathrm{E}-05$ & 2.80 \\
\hline & 2560 & 7.83E-07 & 3.48 & $1.28 \mathrm{E}-06$ & 3.28 \\
\hline \multirow{2}{*}{$\begin{array}{l}\text { Polynomial } \\
\text { order }\end{array}$} & & \multicolumn{2}{|l|}{ "LDG } & \multicolumn{2}{|l|}{ (Our scheme } \\
\hline & \# Elements & $\left\|\nabla u+\mathbf{q}_{h}\right\|_{L^{2}(\Omega)}$ & $\mathrm{RoC}$ & $\left\|\nabla u+\mathbf{q}_{h}\right\|_{L^{2}(\Omega)}$ & RoC \\
\hline \multirow{5}{*}{0} & 10 & $7.98 \mathrm{E}-01$ & & $7.68 \mathrm{E}-01$ & \\
\hline & 40 & $3.12 \mathrm{E}-01$ & 0.83 & 3.89E-01 & 0.98 \\
\hline & 160 & $2.16 \mathrm{E}-01$ & 0.83 & $2.74 \mathrm{E}-01$ & 0.51 \\
\hline & 640 & $1.40 \mathrm{E}-01$ & 0.90 & $1.72 \mathrm{E}-01$ & 0.67 \\
\hline & 2560 & $9.34 \mathrm{E}-02$ & 0.58 & $1.07 \mathrm{E}-01$ & 0.68 \\
\hline \multirow{5}{*}{1} & 10 & $4.57 \mathrm{E}-02$ & & $4.53 \mathrm{E}-02$ & \\
\hline & 40 & 1.62E-02 & 1.50 & $1.47 \mathrm{E}-02$ & 1.62 \\
\hline & 160 & $4.27 \mathrm{E}-03$ & 1.92 & 4.11E-03 & 1.84 \\
\hline & 640 & $1.10 \mathrm{E}-03$ & 1.96 & $1.12 \mathrm{E}-03$ & 1.87 \\
\hline & 2560 & $1.44 \mathrm{E}-04$ & 2.92 & $2.06 \mathrm{E}-04$ & 2.45 \\
\hline \multirow{5}{*}{2} & 10 & $6.99 \mathrm{E}-03$ & & $6.71 \mathrm{E}-03$ & \\
\hline & 40 & $1.11 \mathrm{E}-03$ & 2.66 & $1.11 \mathrm{E}-03$ & 2.59 \\
\hline & 160 & $1.50 \mathrm{E}-04$ & 2.89 & $1.48 \mathrm{E}-04$ & 2.91 \\
\hline & 640 & $1.97 \mathrm{E}-05$ & 2.93 & $1.92 \mathrm{E}-05$ & 2.95 \\
\hline & 2560 & $1.82 \mathrm{E}-06$ & 3.43 & $2.25 \mathrm{E}-06$ & 3.09 \\
\hline
\end{tabular}

Table 7 Unstructured grid test: Errors for primary $\left\|u-u_{h}\right\|_{L^{2}(\Omega)}$ and flux $\left\|\nabla u+\mathbf{q}_{h}\right\|_{L^{2}(\Omega)}$ variables and rates of convergence (RoC).

The type of mixed DG formulation presented in this work is generally wellsuited for hybridized schemes, thus our plans include formulation, evaluation, and analysis of an HDG counterpart to the method presented in this work.

\section{References}

1. Aizinger, V.: A discontinuous Galerkin method for two-and three-dimensional shallowwater equations. Ph.D. thesis, The University of Texas at Austin (2004). URL http://hdl.handle.net/2152/1863

2. Aizinger, V., Dawson, C.: The local discontinuous Galerkin method for threedimensional shallow water flow. Computer Methods in Applied Mechanics and Engineering 196(4), 734746 (2007). DOI 10.1016/j.cma.2006.04.010. URL https://www.math.fau.de/fileadmin/am1/users/aizinger/AizingerDawson2007.pdf

3. Aizinger, V., Dawson, C., Cockburn, B., Castillo, P.: The local discontinuous Galerkin method for contaminant transport. Advances in Water Resources 24(1), 7387 (2000). DOI 10.1016/S0309-1708(00)00022-1 
4. Arnold, D.N., Brezzi, F., Cockburn, B., Marini, L.D.: Analysis of discontinuous Galerkin methods for elliptic problems. SIAM J. Num. Anal. 39, 1749-1779 (2002)

5. Barrios, T.P., Bustinza, R.: An a posteriori error analysis of an augmented discontinuous Galerkin formulation for Darcy flow. Numer. Math. 120(2), 231-269 (2012). DOI 10.1007/s00211-011-0410-3. URL http://dx.doi.org/10.1007/s00211-011-0410-3

6. Brezzi, F., Hughes, T., Marini, L., Masud, A.: Mixed discontinuous Galerkin methods for Darcy flow. Journal of Scientific Computing 22-23, 119-145 (2005). DOI $10.1007 / \mathrm{s} 10915-004-4150-8$

7. Carrero, J., Cockburn, B., Schötzau, D.: Hybridized globally divergence-free LDG methods. Part I: The Stokes problem. Mathematics of Computation 75(254), 533-563 (2006). DOI 10.1090/S0025-5718-05-01804-1

8. Castillo, P., Cockburn, B., Perugia, I., Schötzau, D.: An a priori error analysis of the local discontinuous Galerkin method for elliptic problems. SIAM Journal on Numerical Analysis 38(5), 1676-1706 (2001). DOI 10.1137/S0036142900371003

9. Castillo, P., Cockburn, B., Schötzau, D., Schwab, C.: Optimal a priori error estimates for the hp-version of the local discontinuous Galerkin method for convection-diffusion problems. Mathematics of Computation 71(238), 455-478 (2002). DOI 10.1090/S00255718-01-01317-5

10. Cheng, J., Shu, C.W.: High order schemes for CFD: A review. Jisuan Wuli/Chinese Journal of Computational Physics 26(5), 633-655 (2009)

11. Ciarlet, P.G., Lions, J.L.: Handbook of Numerical Analysis. Elsevier (1990)

12. Cockburn, B., Dawson, C.: Some extensions of the local discontinuous galerkin method for convection-diffusion equations in multidimensions. In: The Proceedings of the Conference on the Mathematics of Finite Elements and Applications: MAFELAP X, p. 225238. Elsevier (2000)

13. Cockburn, B., Shu, C.W.: The local discontinuous Galerkin method for time-dependent convection-diffusion systems. SIAM Journal on Numerical Analysis 35(6), 2440-2463 (1998)

14. Dawson, C.: The $P^{k+1}-S^{k}$ local discontinuous Galerkin method for elliptic equations. SIAM Journal on Numerical Analysis 40(6), 2151-2170 (2002). DOI $10.1137 /$ S0036142901397599

15. Di Pietro, D.A., Ern, A.: Mathematical aspects of discontinuous Galerkin methods. Mathmatiques et applications. Springer, Heidelberg, New York, London (2012)

16. Ern, A., Guermond, J.L.: Theory and practice of finite elements. Applied mathematical sciences. Springer, New York (2004)

17. Hughes, T.J.R., Masud, A., Wan, J.: A stabilized mixed discontinuous Galerkin method for Darcy flow. Comput. Methods Appl. Mech. Engrg. 195(25-28), 3347-3381 (2006). DOI 10.1016/j.cma.2005.06.018. URL http://dx.doi.org/10.1016/j.cma.2005.06.018

18. Huynh, H.: A flux reconstruction approach to high-order schemes including discontinuous Galerkin methods. In: Collection of Technical Papers - 18th AIAA Computational Fluid Dynamics Conference, vol. 1, pp. 698-739 (2007). DOI 10.2514/6.2007-4079

19. Knabner, P., Angermann, L.: Numerical Methods for Elliptic and Parabolic Partial Differential Equations. Springer (2003)

20. Masud, A., Hughes, T.J.R.: A stabilized mixed finite element method for Darcy flow. Comput. Methods Appl. Mech. Engrg. 191(39-40), 4341-4370 (2002). DOI 10.1016/S0045-7825(02)00371-7. URL http://dx.doi.org/10.1016/S00457825(02)00371-7

21. Nguyen, N., Peraire, J., Cockburn, B.: Hybridizable discontinuous Galerkin methods. Lecture Notes in Computational Science and Engineering 76 LNCSE, 63-84 (2011). DOI 10.1007/978-3-642-15337-2_4

22. Peraire, J., Persson, P.O.: The compact discontinuous Galerkin (CDG) method for elliptic problems. SIAM Journal on Scientific Computing 30(4), 1806-1824 (2007). DOI $10.1137 / 070685518$

23. Perugia, I., Schötzau, D.: An hp-analysis of the local discontinuous Galerkin method for diffusion problems. Journal of Scientific Computing 17(1), 561-571 (2002). URL http://dx.doi.org/10.1023/A:1015118613130

24. Reed, H., Hill, T.R.: Triangular mesh methods for the neutron transport equation. Tech. Rep. LA-UR-73-479, Los Alamos Scientific Laboratory, NM (1973) 
25. Shu, C.W.: A brief survey on discontinuous Galerkin methods in computational fluid dynamics. Advances in Mechanics 43(6), 541-554 (2013). DOI 10.6052/1000-0992-13059

26. Shu, C.W.: High order WENO and DG methods for time-dependent convectiondominated PDEs: A brief survey of several recent developments. Journal of Computational Physics 316, 598-613 (2016). DOI 10.1016/j.jcp.2016.04.030

27. Sun, S., Wheeler, M.: Symmetric and nonsymmetric discontinuous Galerkin methods for reactive transport in porous media. SIAM Journal on Numerical Analysis 43(1), 195-219 (2005). DOI 10.1137/S003614290241708X

28. Sun, S., Wheeler, M.: Analysis of discontinuous Galerkin methods for multicomponent reactive transport problems. Computers and Mathematics with Applications 52(5), 637-650 (2006). DOI 10.1016/j.camwa.2006.10.004

29. Thomeé, V.: Galerkin Finite Element Methods for Parabolic Problems (2nd ed.). Springer (2006)

30. Xu, Y., Shu, C.W.: Local discontinuous Galerkin methods for high-order time-dependent partial differential equations. Communications in Computational Physics 7(1), 1-46 (2010). DOI 10.4208/cicp.2009.09.023

31. Zhang, M., Shu, C.W.: An analysis of three different formulations of the discontinuous Galerkin method for diffusion equations. Mathematical Models and Methods in Applied Sciences 13(03), 395-413 (2003). DOI 10.1142/S0218202503002568. URL http://www.worldscientific.com/doi/abs/10.1142/S0218202503002568

32. Zhang, X., Shu, C.W.: Maximum-principle-satisfying and positivity-preserving highorder schemes for conservation laws: Survey and new developments. Proceedings of the Royal Society A: Mathematical, Physical and Engineering Sciences 467(2134), 27522776 (2011). DOI 10.1098/rspa.2011.0153 